\title{
UNA TRAMPA DE DESIGUALDAD \\ Las consecuencias del emparejamiento selectivo en la distribución de ingresos
}

\author{
Estéfano Rubio \\ Centro de Estudios Públicos
}

\begin{abstract}
RESUMEN: Este trabajo busca encontrar cuáles son los efectos del emparejamiento selectivo en educación sobre variables como la desigualdad, los ingresos y el nivel educacional de la sociedad. Con este fin se simula un escenario, para el caso de Chile, en que los padres de los individuos actuales se hubieran emparejado de manera aleatoria, sin pretender a personas de su mismo nivel educativo. Se demuestra que, en tal escenario hipotético, la desigualdad en los ingresos de la ocupación principal de las personas, medida con el índice de Gini, caería desde 0,48 a 0,43 , una baja equivalente a lo que se redujo la desigualdad entre 1990 y 2013 para esta misma variable. Al mismo tiempo, los ingresos, en promedio, se verían disminuidos en 15 por ciento, se reducirían en 5 por ciento las personas sin educación y en 5 por ciento también las personas con educación superior.

Palabras Clave: emparejamiento selectivo, homogamia, desigualdad, movilidad educacional intergeneracional.
\end{abstract}

RECIBIDO: agosto 2016; ACEPTADO: octubre 2016.

Clasificación JEL: D31, I24, J12, J62.

EstÉFAno RuBio. Economista por la Universidad de Chile y magíster en políticas públicas por la misma casa de estudios. Investigador del Centro de Estudios Públicos. Email: erubio@cepchile.cl.

* El autor agradece los comentarios realizados por Harald Beyer, Loreto Cox, Andrés Hernando y Slaven Razmilic, así como los hechos por los árbitros anónimos de Estudios Públicos. 


\title{
AN INEQUALITY TRAP: THE CONSEQUENCES OF ASSORTATIVE MATING ON INCOME DISTRIBUTION
}

\begin{abstract}
This paper looks at the effects of assortative mating on education for variables such as inequality, income and the level of education of society. For Chile, it simulates a scenario where the parents of this generation would have mated randomly, without regard to their mate's level of education. It demonstrates that in that hypothetical scenario, the inequality of household income, measured using the Gini coefficient, would fall from 0.48 to 0.43 , a drop equivalent to the reduction in inequality between 1990 and 2013 (for this same variable). On average, income would simultaneously fall 15 percent, the uneducated would decrease by 5 percent, and the college graduates would also drop 5 percent.
\end{abstract}

Keywords: Assortative mating, homogamy, inequality, intergenerational educational mobility.

ReCEIVED: August 2016; ACCEPTED: October 2016.

Clasification JEL: D31, I24, J12, J62.

\section{INTRODUCCIÓN}

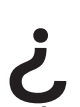

Por qué, a pesar del significativo aumento en la escolaridad que ha tenido Chile, las brechas en ingresos entre los hogares que tienen más y los que tienen menos siguen siendo tan grandes? ¿Será que, a pesar de los esfuerzos de los gobiernos, las personas están tomando decisiones que inhiben el avance en materia de desigualdad? Eso es lo que se pregunta en este trabajo.

A continuación se presenta un análisis del emparejamiento selectivo u homogamia ${ }^{1}$ en educación que posee la sociedad chilena. El estudio devela que los índices de este fenómeno son altos para Chile en comparación con otros países, tanto desarrollados como latinoamericanos. Las principales implicancias de esto son: i) aumento de la desigualdad de ingresos, al provocar que existan familias conformadas por parejas muy educadas y otras en donde ambos integrantes posean muy poca o nula educación. Lo anterior, sumado al alto retorno de la escolaridad, termina generando hogares de muy altos ingresos y otros de un nivel considerablemente inferior. Si este fenómeno coexiste además con

${ }^{1}$ El concepto también se conoce como "homofilia", y se refiere al fenómeno en que ambos integrantes de una pareja tienen atributos similares. Para el caso de este estudio, la escolaridad. 
una baja movilidad educacional intergeneracional y elevados índices de segregación, entonces ii) existiría un contexto bajo el cual se van replicando las estructuras sociales que podrían generar una trampa de desigualdad. Finalmente, iii) ello limitaría el alcance de políticas públicas que apuntan a disminuir la brecha.

Para demostrar lo anterior, este trabajo realiza una simulación que plantea cómo sería hoy la distribución de ingresos y de niveles educacionales si los padres de la generación actual se hubiesen emparejado de forma aleatoria, es decir, sin emparejamiento selectivo. A partir de este análisis, de equilibrio parcial, se muestra que la desigualdad en los ingresos de la ocupación principal de las personas, medida con el índice de Gini, caería desde 0,48 a 0,43. Esta caída de cinco puntos del Gini es equivalente a lo mismo que se redujo la desigualdad entre 1990 y 2013 (para esta misma variable). Por otra parte, los ingresos se verían disminuidos 15 por ciento en promedio; mientras que se reducirían en 5 por ciento las personas sin educación (y/o con educación básica incompleta) y en la misma proporción con educación superior.

Es importante señalar que si bien no se puede rechazar a priori una causalidad inversa (es decir, que mayor desigualdad genere mayores índices de homogamia) o la existencia de otros factores que afecten simultáneamente ambas variables, de todas formas el efecto del emparejamiento selectivo sobre la desigualdad de los ingresos de los hogares es provocado de forma directa (casi por construcción, si se quiere), por lo que en el peor de los casos se podría hablar de la existencia de un círculo vicioso de este fenómeno, mas no se debería obviar su impacto. Es por ello que, a pesar de la naturaleza ficticia de este ejercicio (al asumir un escenario de emparejamiento aleatorio) y de que los resultados puedan ser interpretados sólo como correlaciones, es igualmente interesante observar lo que ocurriría en este contexto.

Este artículo se compone de cinco secciones. Luego de esta introducción, la segunda parte explica la relación existente entre homogamia y movilidad educacional intergeneracional, y los niveles que presenta Chile en estas dos variables durante las últimas décadas, mostrando la correspondiente revisión de literatura. La sección 3 se compone de una explicación de la simulación realizada, una presentación detallada de la metodología y de los resultados. En la sección 4 se presenta un análisis de estos últimos y algunas implicancias de política pública. Finalmente, la sección 5 ofrece una conclusión. 


\section{CONTEXTO}

\subsection{La relación entre emparejamiento selectivo y movilidad educacional Intergeneracional}

Si bien los gobiernos realizan constantes esfuerzos para masificar y distribuir más igualitariamente la educación en la población, en especial en aquellos grupos con menores oportunidades, también existen otros factores determinantes que poseen una naturaleza diferente y que pueden limitar seriamente dichos esfuerzos.

Uno de estos problemas de fondo es el llamado emparejamiento selectivo (assortative mating) u homogamia. Éste consiste en la tendencia que poseen los individuos a relacionarse o formar pareja con personas que poseen características similares a sí mismos, tales como coeficiente intelectual, estatura, atractivo físico, color de piel, origen étnico, religión y, por supuesto, educación (Becker 1973).

Este fenómeno es importante para explicar las desigualdades de ingresos entre los diversos hogares de un país (Breen y Andersen 2012; Eika et al. 2014; Fernández et al. 2005). Mientras mayor assortative mating (AM) haya, más pronunciadas serán las diferencias de ingresos de la sociedad, ya que existirán hogares conformados por parejas de alta educación muy distintos de aquéllos formados por parejas de baja educación, generándose así dos fuentes de altos ingresos en los primeros y dos fuentes de bajos ingresos en los últimos. Esto contrastaría con un escenario contrafactual, en el cual hubiese más familias compuestas por parejas con niveles educacionales heterogéneos. ${ }^{2}$

Otro factor relevante, que eventualmente podría afectar la desigualdad de ingresos y que, además, menoscaba las oportunidades de distintos grupos de la sociedad, es el de la movilidad educacional intergeneracional (en adelante MEI). La MEI corresponde a cuánto cambia el nivel educacional de un hijo comparándolo con el de sus padres. Dicho de otra manera, esta variable puede entenderse como la distancia que existe entre la escolaridad entre progenitores y su descendencia.

${ }^{2}$ Por ejemplo, piénsese en un país que se compone de dos familias, donde quienes tienen alta escolaridad ganan 100 y quienes tienen baja ganan 50. Si hubiese homogamia, entonces habría una familia ganando 200 y la otra 100. El contrafactual, sin homogamia, sería que hubiese dos familias ganando 150. Claramente, el último escenario refleja mayor igualdad de ingresos que el primero. 
De esta manera existirían tres casos: i) MEI $>0$ implicaría que los hijos son más educados que los padres; ii) $\mathrm{MEI}=0$, hijos y padres tienen igual escolaridad o nivel educacional; y iii) $\mathrm{MEI}<0$, los progenitores son más educados. Entonces, a menor MEI, en valor absoluto, el nivel educacional alcanzado por los padres se convierte en un determinante importante del nivel que pueden llegar a alcanzar sus hijos. En otras palabras, si el padre y la madre tuvieron baja escolaridad, entonces es probable que su hijo también la tenga, y viceversa en caso que hayan tenido un nivel educacional alto.

Por las mismas razones, si una misma sociedad posee baja MEI y elevado emparejamiento selectivo, ello podría provocar mayor desigualdad aún de lo que podría generar un alto nivel de homogamia por sí solo.

¿Esto significa que la propensión de una persona a tener educación superior pudiera crecer más al poseer sus dos padres dicha educación versus un escenario en donde sólo uno de ellos la poseyera? Este caso suele ser descartado en la literatura, ya que para analizar la MEI, por ejemplo, suele observarse sólo al progenitor con el mayor nivel educacional (Gaviria 2007; González y Mackenna 2015), omitiendo la información del otro. Esto toma como supuesto, implícitamente, que el aporte marginal del padre con menor escolaridad no debiese influir en el nivel educacional del individuo.

En caso de que este supuesto no se cumpliese, indicaría que en un escenario de baja MEI y bajo emparejamiento selectivo, disminuiría el porcentaje de gente con educación superior así como el porcentaje de gente sin educación, y aumentaría la proporción en niveles intermedios (educación básica y media), haciendo más lepto-kúrtica la distribución. ${ }^{3}$ Ahora, si este fenómeno ocurriese, entonces debiese suceder lo siguiente: al pasar gente de un nivel educacional al siguiente, las ganancias en ingresos, producidas por el aumento de escolaridad de quienes pasan de no tener educación a tener educación básica, serían sustancialmente menores en relación con lo que dejarían de percibir quienes pasasen de

${ }^{3}$ Ello se produciría porque al haber un bajo emparejamiento selectivo aumentaría la heterogeneidad educacional de las parejas. Mientras que si es elevada la herencia educacional entre generaciones de padres a hijos — debido a la baja MEI-, entonces existirá un mayor porcentaje de individuos en esta nueva generación que posea, por un lado, un aumento en su propensión a tener más escolaridad (producto de tener un padre más educado), pero, por el otro, habrá una disminución en esta propensión debido a que el otro progenitor no tendría un nivel educacional igual de elevado, generándose así que más personas acaben por tener niveles educacionales ni tan elevados, ni tan bajos. 
poseer educación superior a educación media (Beyer 2011). En tal caso, entonces la distribución de ingresos se tornaría más simétrica (disminuyendo la asimetría positiva que posee en el caso chileno), provocando en consecuencia que disminuyese la desigualdad. Al mismo tiempo, dado los estimadores de la distribución para Chile, disminuirían los ingresos promedio.

En las siguientes secciones se observará si se cumple o no el supuesto en cuestión, es decir, que el aporte marginal del padre con menor escolaridad no debiese influir en el nivel educacional del individuo. De no cumplirse, querría decir que en el agregado la segregación social, mediante el canal del emparejamiento selectivo de las parejas, pudiese tener costos adicionales en términos de desigualdad, pero ganancias en términos de escolaridad ${ }^{4}$ e ingresos medios.

A continuación, se revisarán los niveles de emparejamiento selectivo y MEI en Chile, comparándolos con los de algunos países desarrollados.

\subsection{Emparejamiento selectivo en Chile}

Como fue adelantado, la homogamia puede terminar siendo un medio de reproducción intergeneracional de la desigualdad. A partir de ello se hace necesario poder cuantificar este fenómeno. Existen diferentes formas de medir el emparejamiento selectivo. Aquí se presentarán dos de éstas.

La primera, que es la más directa y simple, es mediante la correlación de años de escolaridad entre las personas que forman pareja. En el gráfico 1 puede observarse la evolución que esta correlación ha tenido en Chile desde 1990 hasta 2013, donde 1 describe el máximo emparejamiento selectivo posible y 0 , el menor. ${ }^{5}$

Las cifras, si bien han mostrado un leve descenso en el período analizado (pasando de 0,75 en 1990 a 0,72 en 2013), son altas en comparación con otros países. Para Estados Unidos, Warren (1966) encon-

${ }^{4}$ Lo que podría traducirse en que el capital cultural de los hogares no crecería a tasas decrecientes; más bien, el aporte de ambos padres sería igualmente trascendente para la educación de los hijos, y no sólo el del más educado.

${ }^{5}$ Se muestra la correlación calculada sobre todas las parejas, no sólo sobre las parejas de padres de los individuos, como podría pensarse dada la naturaleza de la simulación. 
tró valores cercanos a 0,60, en tanto que Becker (1973) mostró una correlación de 0,53 para blancos y 0,56 para negros. Para Latinoamérica, Valenzuela y Duryea (2011) presentan correlaciones de 0,66 para Argentina en 2002; 0,65 para Brasil y Costa Rica en 2003; 0,67 para Uruguay en el mismo año; y 0,70 para México en 2004. Buss (1985) señala que una variable como la edad al interior de cada pareja posee una correlación entre 0,70 y 0,90 , y que valores mayores a 0,50 ya pueden considerarse como elevados. Por lo tanto, puede decirse con seguridad que la correlación de los años de escolaridad de las parejas en Chile es alta.

\section{Gráfico 1. CORRELACIÓN DE ESCOLARIDAD DE LA PAREJA}

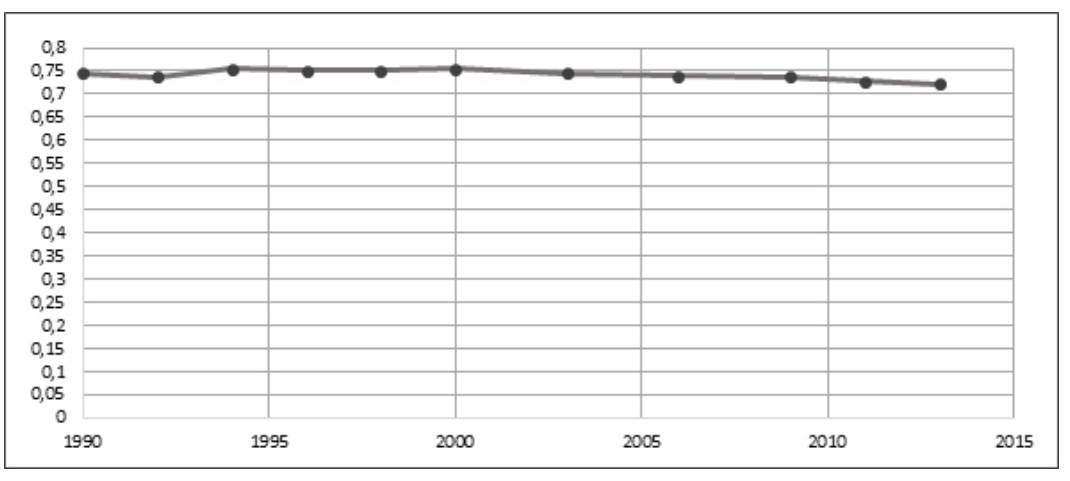

Fuente: Elaboración del autor a partir de datos de la encuesta Casen, años respectivos.

Valenzuela y Duryea (2011) también observan estas correlaciones para Chile en áreas urbanas en los años 1992 y 2003, y registran correlaciones de 0,717 y 0,714 , respectivamente. ${ }^{6}$

No obstante, tal como señala Torche (2010), existen limitaciones ${ }^{7}$ al usar una simple medida agregada de emparejamiento selectivo (como

\footnotetext{
${ }^{6}$ Resultados que coinciden con los de este trabajo al restringir la muestra sólo a la zona urbana.

${ }^{7}$ Por ejemplo, el no permitir observar las fuentes de variación del emparejamiento selectivo, lo que omite también la heterogeneidad existente, para cada nivel educacional, entre distintos países o períodos. Podría haber una evolución distinta del emparejamiento selectivo para los niveles extremos ("Educación superior" y "Sin educación") en relación con los niveles medios ("Educación básica" y "Educación media").
} 
la correlación de años de escolaridad) para capturar la relación entre variación en homogamia y su relación con desigualdad; de igual manera, Siow (2015) también considera la mera correlación como una medida débil. Es por ello que a continuación se presenta otra metodología de cálculo de emparejamiento selectivo que cumple con no ser una medida agregada, pero que no se ha aplicado anteriormente para Chile $^{8}$ y que nos ofrece resultados novedosos.

Una manera alternativa de medir el grado de emparejamiento selectivo de una sociedad es mediante la forma realizada por Eika et al. (2014), quienes miden la clasificación marital entre los niveles educacionales i y j como la probabilidad observada de que un hombre con nivel educacional j esté casado (o emparejado) con una mujer con nivel educacional i, relativo a la probabilidad bajo emparejamiento aleatorio con respecto a la educación:

$$
c_{i j}=\frac{\operatorname{Pr}(\text { Mujer }=i \cap \text { Hombre }=j)}{\operatorname{Pr}(\text { Mujer }=i) * \operatorname{Pr}(\text { Hombre }=j)}
$$

Aquí el numerador corresponde a la probabilidad conjunta observada de que una mujer con nivel educacional i esté emparejada con un hombre de nivel $\mathrm{j}$, y el denominador corresponde al producto entre las probabilidades marginales observadas de la mujer poseyendo nivel i y el hombre nivel $\mathrm{j}$.

Si hombres y mujeres con igual nivel de educación se casasen (o emparejasen) más frecuentemente de lo que sería esperado bajo un patrón aleatorio en términos de educación, ello querría decir que habría un emparejamiento selectivo positivo. Esto se reflejaría en un parámetro de clasificación marital $c_{i j}$ mayor a 1 cuando i $=\mathrm{j}$; y viceversa. Así el parámetro de clasificación marital $\left(c_{i j}\right)$ será mayor a 1 cuando cierta combinación de nivel educacional ocurra con más frecuencia de lo que debería hacerlo en un escenario aleatorio.

Esta medida de homogamia, aplicada de la forma en que aquí se utiliza, permite estudiar patrones de emparejamiento independiente de

${ }^{8}$ Difiere con la empleada por Torche (2010). Esa metodología está basada en un modelo log-lineal de niveles, en donde éstos capturan distintos grados de asociación para cada celda. 
cambios en la oferta poblacional y en las tasas de matrimonio, elementos relevantes a considerar según Siow (2015). Lo primero es tomado en cuenta, ya que el índice utilizado observa los cambios no sólo de la probabilidad conjunta, sino que también de las marginales. Y un cambio en la oferta poblacional, como lo describe Siow, se vería reflejado en ambas. Por otra parte, este trabajo estudia matrimonios y parejas en general también, por lo que cambios en las preferencias por contraer matrimonio no debiesen afectar a este indicador.

En línea con lo anterior, sería deseable exigirle al indicador de emparejamiento selectivo que la medida que proporcione sea independiente de shocks que alteren, dígase, nominalmente, la probabilidad de encontrar una pareja en cierta clasificación de nivel educacional de cada integrante. Por ejemplo, considérese una política que hubiese hecho obligatoria la instrucción primaria, ello generaría a posteriori un aumento de personas con educación básica $\mathrm{y}$, por lo tanto, sería más probable encontrar una pareja en donde ambos tengan dicho nivel. Sin embargo, ello no implicaría necesariamente que el emparejamiento selectivo de la clasificación básica-básica (o sea, aquella en que el hombre y la mujer poseen educación básica) haya aumentado, pues el alza en la probabilidad podría ocurrir por el shock y no por un cambio en las preferencias por emparejarse con personas con el mismo nivel educacional. De esta forma, un indicador fiable de emparejamiento selectivo correspondería a uno que mida el nivel real de éste.

Precisamente, como ya fue analizado, el ratio empleado como indicador (el sistema propuesto por Eika et al.) sí cumple con satisfacer lo que se ha explicado en el párrafo previo, pues ajusta la probabilidad conjunta por las probabilidades marginales de cada integrante de la pareja. En el ejemplo anterior, si bien aumentase el numerador del indicador, al subir la probabilidad de encontrar a un hombre y una mujer en la categoría básica-básica, ello se ajustaría por un aumento también producido en el denominador de la fracción. Pudiendo, de esta forma, incluso disminuir el emparejamiento selectivo real (medido en la variable), si es que la probabilidad de encontrar un hombre o una mujer aumentase más de lo que lo hiciese la probabilidad conjunta. 
La tabla 1 muestra los valores de estos parámetros para cada combinación de nivel educacional para hombres y mujeres que conformaban una pareja, ${ }^{9}$ para los años 1990, 2000 y 2013:

Tabla 1. EVOLUCIÓN DEL EMPAREJAMIENTO SELECTIVO (AM) POR NIVEL EDUCACIONAL. A mayor valor del parámetro, mayor será el emparejamiento selectivo.

1990

\begin{tabular}{|c|c|c|c|c|}
\hline Educ. mujer & Sin educación & Básica & Media & Superior \\
\hline Sin educación & 2,40 & 0,81 & 0,19 & 0,07 \\
\hline Básica & 0,65 & 1,61 & 0,76 & 0,23 \\
\hline Media & 0,14 & 0,51 & 2,21 & 1,93 \\
\hline Superior & 0,09 & 0,27 & 1,15 & 6,22 \\
\hline
\end{tabular}

2000

\begin{tabular}{|c|c|c|c|c|}
\hline Educ. mujer & Sin educación & Básica & Media & Superior \\
\hline Sin educación & 2,98 & 0,87 & 0,21 & 0,03 \\
\hline Básica & 0,78 & 1,70 & 0,76 & 0,23 \\
\hline Media & 0,18 & 0,66 & 1,88 & 1,09 \\
\hline Superior & 0,02 & 0,19 & 0,89 & 4,66 \\
\hline
\end{tabular}

2013

\begin{tabular}{|c|c|c|c|c|}
\hline Educ. mujer & Sin educación & Básica & Media & Superior \\
\hline Sin educación & 3,41 & 1,06 & 0,34 & 0,05 \\
\hline Básica & 1,02 & 1,82 & 0,76 & 0,16 \\
\hline Media & 0,25 & 0,74 & 1,61 & 0,90 \\
\hline Superior & 0,05 & 0,17 & 0,75 & 3,61 \\
\hline
\end{tabular}

Fuente: Elaboración del autor a partir de datos de la encuesta Casen, años respectivos.

${ }^{9}$ Es importante mencionar que, para poder identificar a las parejas existentes, se consideró sólo a los núcleos de hogares que reportaban estar casados o estar en parejas heterosexuales (las razones para no incluir a las parejas del mismo sexo son: (i) carecen de apropiada representatividad estadística en este tipo de encuestas; (ii) para que el estudio posea comparabilidad con el resto de la literatura, que tampoco analiza parejas del mismo sexo). Es decir, el análisis no incluye a posibles parejas compuestas por dos integrantes de núcleos diferentes. Lo cual también es positivo, pues dicho análisis toma en cuenta sólo relaciones ya establecidas, es decir, se controla por aquellas que pudiesen tener horizontes de corto plazo que no llegan a determinar la composición de ingresos de hogares. Además, tal como señala Torche (2010), a diferencia de otros trabajos acerca del emparejamiento selectivo que analizan sólo matrimonios, es importante considerar también a las parejas que conforman hogares (para el caso de Chile). Según el Censo del 2002, el 18 por ciento del total de parejas que conforman un hogar no se encuentran casadas. 
Puede compararse estos resultados con los de Eika et al. (2014) que se muestran en la tabla 2. Ellos aplicaron el mismo análisis ${ }^{10}$ a Estados Unidos y Noruega para los años 1980 y 2007 . De ese trabajo se nota que ambos países tienen un emparejamiento selectivo positivo, aunque considerablemente menor que Chile (a excepción de Estados Unidos para la categoría de "educación media incompleta", aunque no es directamente comparable con las categorías analizadas para Chile). En especial merece observarse con detenimiento la categoría $\mathrm{i}=\mathrm{j}=$ "educación superior". Ésta muestra un parámetro $c_{i j}$ cercano a 2 para Estados Unidos y Noruega, mientras que en Chile dicho valor es casi el doble. Esta cifra puede tener consecuencias muy grandes a la hora de mirar la desigualdad del país, en especial tomando en cuenta el alto retorno a la educación superior que existe en Chile (Beyer 2011). Sin embargo, es interesante notar que, para el período de 27 años estudiado por los autores, en comparación con el de 23 que se presenta en la tabla 1, existe una tendencia muy similar en el emparejamiento selectivo: se observa una reducción para los índices de clasificaciones de mayor escolaridad ("media" y "superior") y un incremento en los de menores niveles educacionales ("sin educación” y "básica"). Es posible pensar que esta tendencia tenga alguna relación con la política que estableció la educación media obligatoria y con la masificación de la educación superior, sin embargo, como ya se señaló, el que el indicador controle por las probabilidades marginales permite plantear reparos a esta hipótesis.

En el gráfico 2 se puede observar un despliegue de los datos utilizados en la tabla 1 para el año 2013 en Chile, donde destacan los peaks de la homogamia para las categorías, donde $\mathrm{i}=\mathrm{j}=$ "sin educación" $\mathrm{e} \mathrm{i}=$ $\mathrm{j}=$ "educación superior", es decir, en los casos en que ambos integrantes de la pareja poseen dichos niveles educacionales.

Para poder explicar la tendencia según la cual disminuye el emparejamiento selectivo de los individuos más educados y aumenta el de los menos educados, existen dos hipótesis. Por un lado, puede que se haya incrementado el nivel de homogamia o assortative mating de las categorías correspondientes, o puede deberse a cambios en la distribución de la educación. Observando los resultados expuestos en la tabla 3 puede apreciarse que es probable que no se deba a la primera hipótesis, puesto

${ }^{10}$ Además de que estos autores analizaron exclusivamente parejas casadas, las categorías de los niveles educacionales son lo único en que levemente difiere el análisis. Ellos utilizan "educación media incompleta", "educación media completa", "educación superior incompleta" y "educación superior completa". 
8

บ
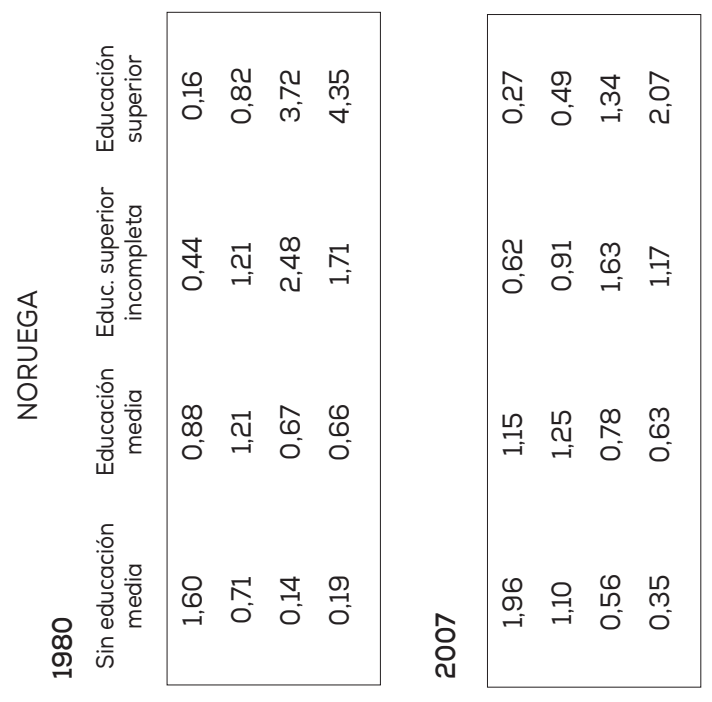

중

๑

o

品

을

过

Ш

志

o

文

응

过

늘

岁

ऐ

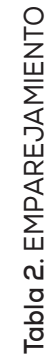
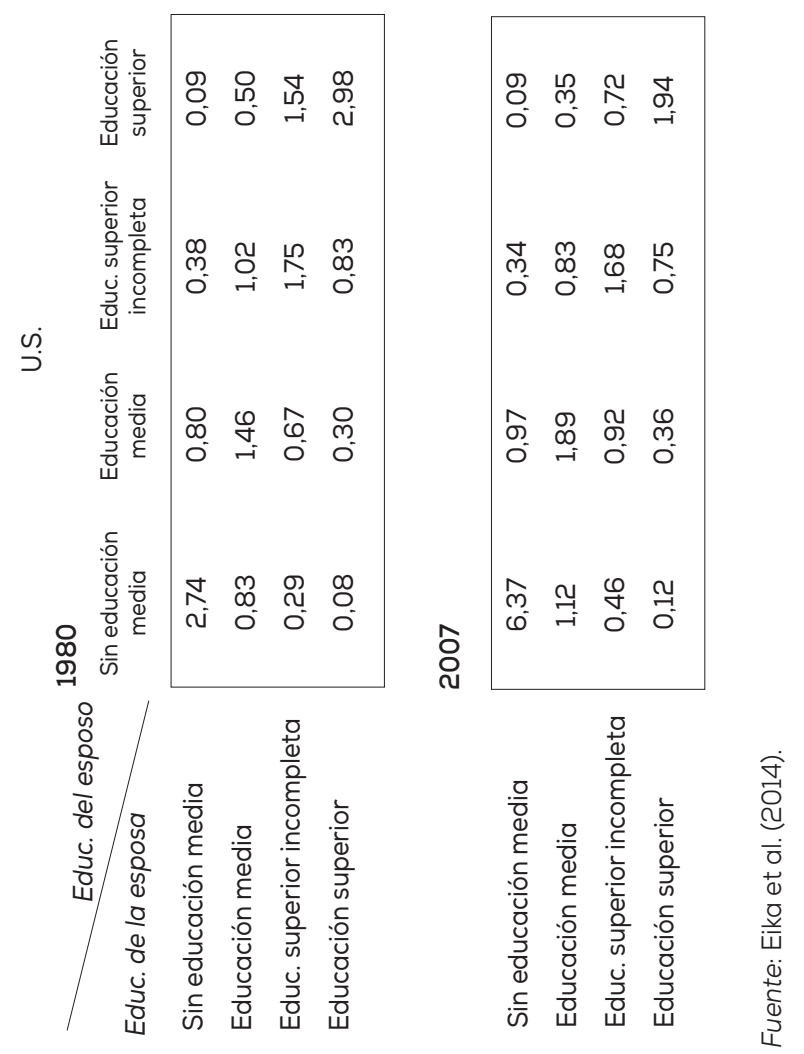
Gráfico 2. EMPAREJAMIENTO SELECTIVO POR NIVELES EDUCACIONALES PARA EL AÑO 2013

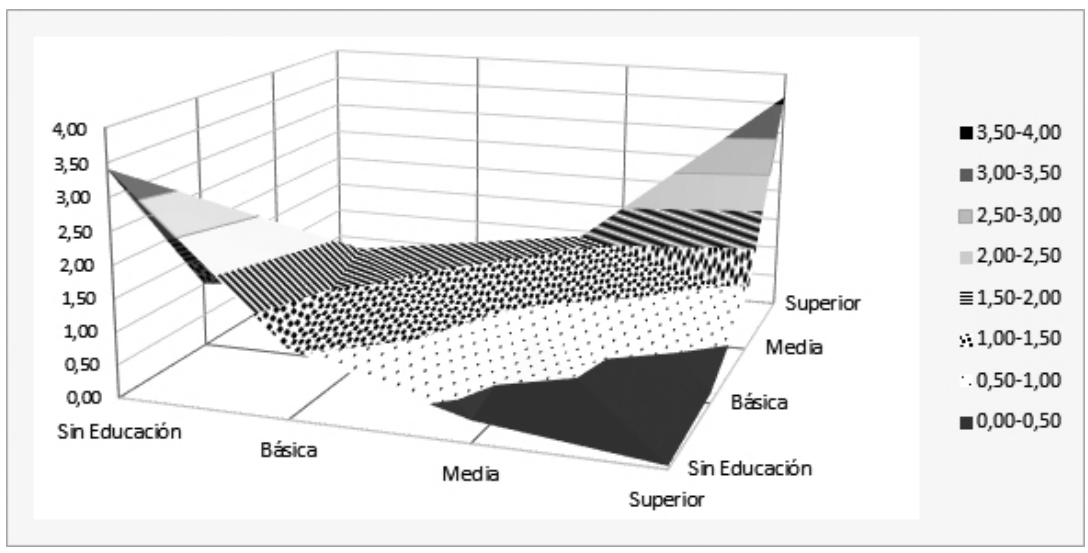

Fuente: Elaboración del autor a partir de datos de la encuesta Casen 2013.

que el nivel de emparejamiento selectivo ponderado ${ }^{11}$ se ha mantenido bastante similar en el período analizado, tal como ocurre con Estados Unidos y Noruega, aunque, como se ve en la tabla, se aprecia una leve disminución por parte de Chile y un leve aumento frente a los primeros. En síntesis, la tendencia a que disminuya el emparejamiento selectivo de los individuos más educados y aumente el de los menos se debería a cambios en la distribución de educación de hombres y mujeres, tal como lo confirman Eika et al. (2014).

A pesar de los elevados índices de emparejamiento selectivo que posee Chile, y que son un diagnóstico interesante acerca de por qué no ha progresado más rápido en disminuir la desigualdad, el país ha mostrado una reducción importante en el período analizado para algunas de las clasificaciones. Disminuyó su emparejamiento selectivo para el segmento superior-superior en 42 por ciento, mejor que la baja de Estados Unidos de 34,9 por ciento, aunque un poco inferior a la de Noruega, de 52,4 por ciento, si bien lo logró en un período más breve. Sin embargo, es preocupante el aumento en el parámetro de clasificación $c_{i j}$ de parejas

${ }^{11}$ Éste se calcula sumando la ponderación entre $c_{i=j}$ y la cantidad de personas presentes en dicha categoría, y dividiendo el resultado final por el total de individuos que hay en la diagonal. 
con bajos niveles educacionales, más aún si es que en Chile existen bajos niveles de movilidad educacional intergeneracional. En la siguiente sección se revisará este tema.

Tabla 3. MEDIDA AGREGADA DE EMPAREJAMIENTO SELECTIVO

\begin{tabular}{l|cc|cc|cc}
\hline & \multicolumn{2}{|c|}{ Estados Unidos } & \multicolumn{2}{c|}{ Noruega } & \multicolumn{2}{c}{ Chile } \\
\hline & 1980 & 2007 & 1980 & 2007 & 1990 & 2013 \\
\hline $\begin{array}{l}\text { Medida agregada de } \\
\text { emparejamiento selectivo* }\end{array}$ & 1,93 & 1,97 & 1,45 & 1,55 & 2,37 & 2,27 \\
\hline
\end{tabular}

Fuente: Eika et al. (2014) para Estados Unidos y Noruega; elaboración del autor a partir de la encuesta Casen, años respectivos, para Chile.

*Nota: Esta medida se calcula a partir del promedio ponderado de parámetros de clasificación (Cij) de la diagonal de la matriz de combinaciones de niveles educacionales de la pareja.

\subsection{Movilidad educacional intergeneracional en Chile}

Existen diversas formas de medir la movilidad intergeneracional de los individuos, donde quizás las más usadas sean la de ingresos y la educacional. Para Chile, existen principalmente estudios relativos a la primera (Núñez y Miranda 2007; Torche 2005), sin embargo, son más escasos para la segunda.

Para analizar la movilidad educacional intergeneracional (MEI) se estimó por Mínimos Cuadrados Ordinarios (MCO), una regresión donde la variable dependiente fue el nivel educacional ${ }^{12}$ de la persona y como variable explicativa relevante se usó el nivel educacional ${ }^{13}$ más

${ }^{12}$ Los niveles utilizados son: sin educación, básica, media, técnica nivel superior y universitaria nivel superior.

${ }^{13}$ La razón de usar niveles en lugar de años de escolaridad estriba en que: i) en la encuesta Casen 2006 sólo se pregunta por el nivel educacional de los padres y no el detalle de los años de escolaridad; así, por motivos de comparabilidad temporal, se optó por la primera alternativa en todos los casos; ii) los individuos recuerdan (y por ende reportan) con mayor precisión su escolaridad en niveles en lugar de años específicos; y, por último, iii) el efecto de la educación no es continuo en los años de escolaridad. Es importante agregar que, sólo por motivos de comparación con los trabajos mencionados a continuación se optó por presentar los resultados por MCO y no los de un modelo ordenado (probit o logit ordenado), que hubiese sido lo más apropiado para esta estimación en donde los valores que puede tomar la variable dependiente son pocos (los niveles educacionales). 
alto alcanzado por alguno de los padres. ${ }^{14}$ Esta metodología también ha sido aplicada por Gaviria (2007) y González y Mackenna (2015). También se agregaron algunos controles, ${ }^{15}$ que se pueden observar en la tabla 4 , junto con los resultados de la estimación.

Tabla 4. ESTIMACIÓN DEL NIVEL EDUCACIONAL DE CADA INDIVIDUO CON RESPECTO AL DE SUS PADRES

\begin{tabular}{lrrrr}
\hline Variables & 2006 & 2009 & 2011 & 2013 \\
\hline $\begin{array}{l}\text { Nivel educacional de } \\
\text { los padres }\end{array}$ & 0,4706 & 0,5160 & 0,5196 & 0,5094 \\
Sexo (Hombre = 1) & 0,1092 & 0,0976 & 0,1060 & 0,0871 \\
Edad & 0,0180 & 0,0328 & 0,0304 & 0,0357 \\
Edad & $-0,0003$ & $-0,0005$ & $-0,0004$ & $-0,0005$ \\
Tiene pareja (Si = 1) & 0,0404 & 0,0583 & 0,0861 & 0,0737 \\
Zona (Urbano = 1) & 0,4899 & 0,4912 & 0,4996 & 0,4865 \\
Número de personas & $-0,0522$ & $-0,0553$ & $-0,0494$ & $-0,0589$ \\
en el hogar & & & & \\
\hline Tamaño de la muestra & 98.480 & 82.897 & 65.074 & 68.701 \\
R2 & 0,346 & 0,357 & 0,355 & 0,364 \\
\hline
\end{tabular}

Fuente: Elaboración del autor a partir de la encuesta Casen, años respectivos.

Nota: La variable dependiente es el nivel educacional del individuo. Todos los coeficientes son significativos al 0,1 por ciento.

Según Gaviria (2007), para países desarrollados la correlación entre el nivel de escolaridad de la persona y el de sus padres se ubica entre 0,3 y 0,2 . En sus estimaciones para Latinoamérica, en tanto, encuentra un coeficiente de 0,442. Por su parte, González y Mackenna (2015) ${ }^{16}$

${ }^{14}$ Dado que es sumamente improbable que el nivel educacional de los hijos pudiese modificar el de los padres (puede eventualmente pensarse que un padre podría motivarse por entrar a la universidad luego de que su hijo lo hiciera, lo cual sería raro por varias razones, principalmente por una asimetría etaria con el proceso), entonces podría afirmarse, con bastante seguridad, que la educación de los padres, como predictor de la de los hijos, sería una variable exógena. Lo cual permite la consistencia del estimador por MCO.

15 Todos con los signos esperados.

${ }^{16}$ Ellos utilizaron los datos de la encuesta del Centro de Estudios Públicos del año 2014. 
encuentran un coeficiente de 0,622 para Chile (sin incluir controles en su estimación). Replicando su estimación con los datos de la Casen 2013, y sin incluir controles, se pudo observar que el coeficiente alcanza un valor de 0,587 , una cifra bastante similar a la hallada por estos últimos autores.

A partir de las estimaciones ${ }^{17}$ presentadas en la tabla 4 , se observa que a fines de la década de los 2000 disminuyó la MEI (aumentó su coeficiente). Posteriormente, en 2013, se aprecia un pequeño aumento de la movilidad, aunque se mantiene en niveles inferiores a los registrados en la primera observación en 2006.

Estos resultados sugieren que, bajo una perspectiva internacional, Chile posee una baja MEI, lo cual es preocupante. Esto describe un escenario que no sólo menoscaba la meritocracia, sino que también es ineficiente, pues dificulta que personas con talento salgan adelante. Por otro lado, además de la desigualdad de oportunidades generada entre distintos sectores socioeconómicos de la población, esto también produce un terreno adverso para la disminución de las desigualdades de ingresos de la sociedad al sumarse con los elevados índices de emparejamiento selectivo que posee el país.

\section{SIMULACIONES}

\subsection{Simulando un contrafactual}

A continuación, se presenta un análisis novedoso que, al menos hasta donde alcanza el conocimiento del autor, no ha sido realizado previamente en la literatura y que trata acerca de cómo cambia el índice de Gini ${ }^{18}$ cuando se simula un escenario en el cual no hay emparejamiento selectivo u homogamia en escolaridad por parte de los padres de los individuos de la muestra. Ello permitirá predecir un nuevo nivel de escolaridad para cada persona, con el cual se predecirán nuevos ingresos, asumiendo que las condiciones actuales de transmisión de la educación

${ }^{17}$ Las estimaciones presentadas en este trabajo fueron hechas desde el año 2006 en adelante, debido a que sólo desde ese año se comenzó a preguntar por la educación de los padres del entrevistado en la encuesta Casen.

${ }^{18}$ En esta parte se trabajará con el Gini a nivel individual y no de hogares; más adelante se comprenderán las razones. Éste, como su nombre lo indica, se calcula a partir del ingreso personal y no mediante el promedio del hogar. 
se mantuvieran y que, también, se conservara la relación entre ingresos y niveles educacionales. A partir de este tratamiento, se generará una nueva distribución desde la cual se compararán los niveles de desigualdad. Los resultados de interés finalmente serán la nueva distribución por niveles educacionales, el nuevo ingreso promedio, el Gini y el ratio $10 / 10^{19}$.

Esta simulación resulta ser similar en espíritu, pues tiene la misma finalidad, a la realizada por Greenwood et al. (2014), pues también ellos intentaron comparar la desigualdad actual con un escenario de emparejamiento aleatorio, sin embargo, no sólo lo hacen para un contexto diferente, sino que además usan una metodología distinta a la empleada en este trabajo, como se verá en el siguiente apartado. En particular, destaca en el presente análisis el hecho de hacer la simulación con respecto a los padres de los actuales individuos y no sobre los individuos mismos, porque ello permitirá dos cosas: i) hacer un análisis representativo de toda la población, pues todos los individuos necesariamente tuvieron padres y por ende se observaría una manifestación de los efectos del emparejamiento selectivo a partir de cada persona. ${ }^{20} \mathrm{Si}$, en cambio, se intentara simular cómo sería una situación sin homogamia para las parejas actuales, como lo hacen Greenwood et al. (2014), ello sólo aplicaría a personas que se encontrasen emparejadas. Por lo tanto, el análisis de las implicancias del emparejamiento selectivo en la desigualdad no permitiría observar los efectos que tiene este fenómeno sobre toda la población, sino que sólo sobre los casados o emparejados.

Además, hacer la simulación con respecto a los padres permite ii) observar la validez de la hipótesis según la cual la propensión de los individuos a tener mayores niveles educacionales aumenta a una tasa marcadamente decreciente con el nivel educacional del otro padre. Esto

19 Tal como el Gini, el ratio 10/10 es otra medida de desigualdad que indica la razón existente entre los ingresos promedios del decil más alto y más bajo.

${ }^{20}$ Esto ocurre porque las distintas combinaciones de niveles educacionales de los padres permiten predecir diferentes niveles educacionales de los individuos y, a su vez, ingresos. Por lo tanto, al tomar en cuenta que todos los individuos poseen ingresos y una cierta escolaridad (incluso si ambas fuesen nulas, eso ya reflejaría cierto nivel), entonces las consecuencias del emparejamiento selectivo de la generación de padres afectará directamente en la desigualdad calculada sobre toda la distribución de ingresos. De esta manera, las implicancias del emparejamiento selectivo podrían observarse en toda la población y no sólo en las actuales parejas o matrimonios. 
sería posible debido a que en la predicción del nivel educacional de los individuos también se observaría el efecto de la movilidad educacional intergeneracional. La hipótesis en cuestión se validaría en caso de que se evidencie un aumento de la gente con mayores niveles educacionales. $Y$ es que en dicho caso querría decir que sería más beneficioso, en el agregado, distribuir a los padres más educados con parejas menos educadas, puesto que en total se ganaría mayor propensión a tener más escolaridad.

Antes de explicar en detalle el procedimiento de la simulación, es importante señalar que, tal como ya se vio, incluso países desarrollados poseen cierto nivel de emparejamiento selectivo, por lo que estos resultados simplemente muestran un escenario $-o$ nivel de desigualdad potencial- en el que Chile podría haberse encontrado en caso de haber sido una sociedad menos segregada y más inclusiva. De esta forma se busca mostrar la relevancia de la homogamia.

\subsection{Metodología}

Para entender con mayor claridad la metodología utilizada en este trabajo, ésta se pondrá en contraste con el análisis de Greenwood et al. (2014), en donde ellos señalan que $g_{k}$ denota la fracción de hogares que son del tipo $k$, donde los puede haber compuestos por solteros de distintos niveles educacionales y por parejas de distintas combinaciones de dichos niveles, y $w_{k}$ corresponde al ingreso de dicho tipo de hogar. Luego, si $P$ representa el conjunto de parejas y $S$ el de solteros, entonces, el experimento de estos autores trata de reemplazar los $\left\{g_{k}\right\}$ observados, para cada $k \in P$, con el conjunto que se obtendría de un emparejamiento aleatorio, denotado por $\left\{\tilde{g}_{k}\right\}, \forall k \in$ $P$. Así, el Gini contrafactual quedaría definido por $\operatorname{Gini}\left(\left\{g_{k}^{\prime}\right\},\left\{w_{k}\right\}\right)$, donde $\left\{g_{k}^{\prime}\right\} \equiv\left\{\tilde{g}_{k}\right\}_{P} \cup\left\{g_{k}\right\}_{S}$.

Dado que el objetivo es dimensionar el alcance y las consecuencias que tiene el emparejamiento selectivo sobre toda la sociedad (además de observar cómo media entre el nivel de desigualdad y la escolaridad de la población), entonces el análisis realizado por los anteriores autores es limitado, pues $\left|\operatorname{Gini}\left(\left\{g_{k}^{\prime}\right\},\left\{w_{k}\right\}\right)-\operatorname{Gini}\left(\left\{g_{k}\right\},\left\{w_{k}\right\}\right)\right|$ estaría siendo subestimado debido a que el subconjunto $\left\{g_{k}\right\}_{S}$ permanece inalterado. 
Por lo tanto, dado que por definición $\left\{g_{k}\right\}_{S}$ permanecerá invariante bajo el ejercicio de aleatorizar las parejas, entonces, si se mantienen constante los $\left\{g_{k}\right\}$ observados, se puede proceder a estimar un nuevo conjunto de ingresos $\left\{\hat{\mathrm{w}}_{k}\right\}$, tal que se sabe que $w_{k}\left(\operatorname{esc}_{k l}\left(\operatorname{escp}_{k l m}\right)\right)$; donde $e c_{k l}$ corresponde a la escolaridad del individuo $l$ del tipo de hogar $k \mathrm{y}$ $e c_{k l m}$ es la escolaridad del progenitor $m$ (padre y madre) del individuo $l$ del tipo de hogar $k$. Luego se define a $f_{k}$ como la fracción de hogares de los padres de los individuos que son del tipo $k$. De esta forma, si se aplica el emparejamiento aleatorio sobre los padres, se obtendrá $\left\{\tilde{f}_{k}\right\} \forall k .{ }^{21}$ Con ello se obtendrá un nuevo conjunto $\left\{\widetilde{e s c}_{k l m}\right\}$, lo que permitirá estimar nuevos niveles de escolaridad y salarios $\hat{\mathrm{w}}_{k}\left(\widehat{\operatorname{esc}}_{k l}\right) \forall k$, l. Con lo que el Gini contrafactual quedaría definido por $\operatorname{Gini}\left(\left\{g_{k}\right\},\left\{\hat{\mathrm{w}}_{k}\right\}\right)$, donde $\left\{\hat{\mathrm{w}}_{k}\right\} \equiv$ $\left\{\hat{\mathrm{w}}_{k}\right\}_{P} \cup\left\{\hat{\mathrm{w}}_{k}\right\}_{S}$. De aquí se aprecia que si bien la aleatorización se aplicó sólo en $\left\{f_{k}\right\}$, el hecho de que todos los individuos de la generación actual hayan tenido padres, asegura que los efectos de la aleatorización generen un cambio en toda la distribución de ingresos de la actualidad, y no sólo sobre quienes hoy se encuentren en pareja.

Para realizar el ejercicio descrito se utilizaron los datos de la encuesta Casen del año 2013. Ésta incluye preguntas acerca de la educación de los padres de las personas encuestadas. ${ }^{22}$ No obstante, sólo 68.947 de un total de 218.491 individuos reportaban el nivel educacional de sus dos progenitores. Lo anterior no hubiese sido un inconveniente de no ser porque la distribución de ingresos y de niveles educacionales de los individuos era considerablemente distinta entre la submuestra y la muestra total, lo cual puede verse en la tabla 5 .

Allí se presentan los indicadores de interés mencionados al inicio de esta sección: medidas de desigualdad como índice de Gini y el ratio 10/10, características de la distribución de ingresos (incluyendo promedio y desviación estándar) y la distribución de los niveles educacionales. Los contrastes entre la muestra original y la submuestra son nítidos. Se podría presumir que las diferencias en la distribución de ingresos son producto de las diferencias existentes en la distribución por niveles educacionales. Se observa que en la submuestra de las personas que

${ }^{21}$ Nótese que aquí es $\forall k$ dado que $f_{k}$ se definió específicamente como la fracción de hogares de los padres del tipo $k$, pero se asume que todos los padres forman parte del conjunto de parejas, por ende se cumple que $f_{k}=\left\{f_{k}\right\}_{P}$.

${ }^{22}$ Nótese que las preguntas relativas al nivel educacional de los padres no fueron realizadas solamente al jefe de hogar. 
informaron el nivel educacional de sus padres hay una sobrerrepresentación de las personas con mayor educación y una subrepresentación de quienes no poseen educación y educación básica incompleta. Dado esto, se procedió a realizar imputaciones a la muestra total, con el fin de corregir este problema de representación.

Tabla 5. COMPARACIÓN DE DISTRIBUCIÓN DE INGRESOS Y DE NIVELES EDUCACIONALES DE MUESTRA ORIGINAL Y SUBMUESTRA*

\begin{tabular}{l|ccc}
\hline & & Muestra original & Submuestra \\
\hline \multirow{4}{*}{$\begin{array}{l}\text { Ingreso de la } \\
\text { ocupación principal }\end{array}$} & Gini & 0,48 & 0,51 \\
& Ratio 10/10 & 6,25 & 7,333 \\
& Promedio & 501.020 & 592.710 \\
& Mesviación estándar & 775.985 & 935.981 \\
& Mínimo & 2.270 & 2.270 \\
& Máximo & 43.500 .000 & 43.500 .000 \\
\hline \multirow{3}{*}{ Nivel educacional } & Sin educación (\%) & 31,49 & 15,21 \\
& Educación básica (\%) & 24,40 & 25,29 \\
& Educación media (\%) & 31,76 & 37,94 \\
& Educación superior (\%) & 12,35 & 21,56 \\
\hline
\end{tabular}

Fuente: Elaboración del autor en base a encuesta Casen 2013.

*Nota: La submuestra se limita a las personas que informaron el nivel educacional de ambos padres.

Dicho proceso fue llevado a cabo otorgando a los padres de cada individuo niveles de educación semejantes $-\mathrm{o}$ análogos - a los del grupo que sí informó sobre sus padres, respetando el mapa de emparejamiento selectivo obtenido para cada nivel educativo de esta submuestra. Primero se analizó la distribución de las clasificaciones educacionales $c_{i j}$ (las mismas expuestas en la sección anterior) de los padres de los encuestados, por cada nivel educacional de estos últimos. Por ejemplo, se tomaron a todos los individuos con educación media y se observó el porcentaje de personas que tenían sus padres con cada nivel de clasificación $i j$. Luego, para todos los individuos que también tenían educación media pero que no reportaban el nivel educacional de alguno de sus padres se procedió a imputárseles aleatoriamente un nivel de escolaridad de sus progenitores, de forma tal que se conservasen los 
porcentajes de representatividad de cada clasificación ij de acuerdo a los niveles originales; es decir, se conservaron las distribuciones intra nivel educacional. ${ }^{23}$ Este procedimiento ${ }^{24}$ fue realizado por separado para individuos que reportaban sólo la escolaridad del padre, sólo la de la madre y para quienes no reportaban ninguna, conservando en todos los casos, y también a nivel global, las proporciones originales por nivel educacional de los encuestados.

De haberse optado sólo por mantener las proporciones globales de las clasificaciones $i j$ originales, hubiese habido mayores probabilidades de sesgar los datos en las imputaciones, ya que dichas proporciones, como explicamos, provenían de una submuestra que sobrerrepresentaba los niveles altos de educación y subrepresentaba los bajos. En tanto, al mantener las distribuciones relativas para cada clasificación educacional de los padres dentro de cada nivel educacional de los encuestados, el supuesto de identificación sería menos restrictivo, pues se asumiría que las proporciones de quienes reportaron la educación de ambos padres habrían de ser las mismas que las proporciones poblacionales, pero controlando por el nivel educacional de cada individuo. De todas formas, más adelante se presentarán simulaciones con y sin imputaciones.

Con posterioridad a las imputaciones, se analizó cómo se distribuían las parejas de padres clasificados por sus niveles educacionales, lo que se puede observar en la tabla 6. Con ello se calculó los pará-

${ }^{23}$ Siguiendo el ejemplo mencionado, podía tenerse que a partir de los datos observados se veía que el seis por ciento de las personas con educación media poseían un padre sin educación y una madre con educación básica; mientras que para alguien con educación superior dicho porcentaje correspondía sólo al cuatro por ciento. De esta forma, si había un individuo con educación media pero que no reportaba la educación de alguno de sus progenitores, entonces con probabilidad 0,06 se le asignaría padres con el nivel de clasificación en cuestión, mientras que, si tenía educación superior, se le asignaría esa condición con probabilidad 0,04.

${ }^{24}$ Es importante señalar que, dado que los padres de los entrevistados pertenecen a distintas cohortes, es de esperar que padres más jóvenes posean mayor educación que los progenitores mayores. Si bien éste también podría haber sido un criterio relevante a tomar en cuenta para las imputaciones, se consideró que era más trascendente la sobrerrepresentación de las personas con educación superior y subrepresentación de las personas sin educación en la submuestra; por lo tanto, las imputaciones se realizaron para hacerse cargo de este último inconveniente. Si bien en teoría era factible realizar las imputaciones considerando ambos criterios (e incluso quizás otros más), dicho procedimiento no se efectuó por mera simplicidad del análisis. El proceso de imputación se hubiese complejizado de sobremanera, conforme se añadieran más criterios de representatividad poblacional. 
metros de clasificación $c_{i j}$ como medida de emparejamiento selectivo, según muestra la tabla 7. Es importante mencionar que conservar las proporciones intra nivel educacional no garantiza que se preserven los mismos niveles de assortative mating globales para los padres. En efecto, cambian. En las tablas A.1 y A.2 (ver Anexos), se pueden observar los mismos análisis de las 6 y 7 , pero para la muestra restringida sin imputaciones. ${ }^{25}$

Tabla 6. DISTRIBUCIÓN PORCENTUAL POR NIVEL EDUCACIONAL DE LA EDUCACIÓN DE LOS PADRES DE LOS ENCUESTADOS, ${ }^{26}$ POSTIMPUTACIONES

\begin{tabular}{|l|rrrrr|}
\hline Educ. madre & $\begin{array}{c}\text { Sin educa- } \\
\text { ción }\end{array}$ & Básica & Media & Superior & Total \\
\hline Sin educación (\%) & 53,20 & 6,68 & 1,99 & 0,24 & 62,10 \\
Básica (\%) & 4,67 & 14,63 & 3,28 & 0,60 & 23,19 \\
Media (\%) & 1,19 & 2,13 & 7,93 & 1,25 & 12,50 \\
Superior (\%) & 0,07 & 0,21 & 0,55 & 1,37 & 2,20 \\
Total (\%) & 59,13 & 23,65 & 13,75 & 3,47 & 100,00 \\
\hline
\end{tabular}

Fuente: Elaboración del autor en base a encuesta Casen 2013.

Tal como se indicó con anterioridad, si $c_{i j}=1$, ello indica que no existiría emparejamiento selectivo (es decir, hombres y mujeres de igual nivel de educación no estarían ni más, ni menos frecuentemente emparejados de lo que lo estuvieran mediante un patrón que fuese aleatorio en términos de educación). Entonces, el interés está en conocer cuál sería el valor de $\operatorname{Pr}($ Mujer $=i \cap$ Hombre $=j)$ para todas las combinaciones de i y j, tal que permita obtenerse un ratio de 1 en el indicador $c_{i j}$. Esto ya que, con esas proporciones, al ponderarlas por el número total de observaciones de parejas de padres, permitiría saber cómo sería la distribución por nivel educacional de los padres que sea acorde a un escenario de emparejamiento aleatorio.

${ }^{25}$ En las tablas 6 y 7 se muestra, por ejemplo, que 14,63 por ciento de las personas contaban con ambos padres con educación básica y que esto correspondía a un ratio de 2,67, el cual señala qué tanto más probable, de lo que hubiese sido en un escenario aleatorio, es haber tenido ambos padres con dicha educación.

${ }^{26}$ Estos datos no incluyen factores de expansión, debido a que su utilidad se centra en la construcción del escenario contrafactual. 
Tabla 7. EMPAREJAMIENTO SELECTIVO DE LOS PADRES DE LOS ENCUESTADOS, ${ }^{27}$ POST IMPUTACIONES

\begin{tabular}{|c|c|c|c|c|}
\hline Educ. madre & Sin educación & Básica & Media & Superior \\
\hline Sin educación & 1,45 & 0,45 & 0,23 & 0,11 \\
\hline Básica & 0,34 & 2,67 & 1,03 & 0,75 \\
\hline Media & 0,16 & 0,72 & 4,61 & 2,89 \\
\hline Superior & 0,05 & 0,41 & 1,81 & 18,00 \\
\hline
\end{tabular}

Fuente: Elaboración del autor en base a encuesta Casen 2013.

Ahora, dado que se sabe que:

$$
c_{i j}=\frac{\operatorname{Pr}(\text { Madre }=i \cap \text { Padre }=j)}{\operatorname{Pr}(\text { Madre }=i) * \operatorname{Pr}(\text { Padre }=j)}
$$

Entonces si $c_{i j}=1$ :

$$
\operatorname{Pr}(\text { Madre }=i \cap \text { Padre }=j)=\operatorname{Pr}(\text { Madre }=i) * \operatorname{Pr}(\text { Padre }=j)
$$

Y dado que las probabilidades marginales para el padre y la madre son conocidas a partir de los datos de la tabla 6, entonces puede construirse una distribución benchmark que reflejaría un emparejamiento aleatorio perfecto o completo de los padres de los individuos encuestados. Con este insumo, puede realizarse una comparación entre dicha distribución y la distribución de emparejamiento de los padres aleatorizada computacionalmente. ${ }^{28}$ Así, mientras más pequeñas sean las diferencias entre ambas, más se podrá confiar en la aleatorización realizada. La distribución benchmark ${ }^{29}$ se presenta en la tabla 8, en don-

${ }^{27}$ Resulta sumamente interesante revisar cómo cambió el nivel de emparejamiento selectivo desde esta generación de "padres" en comparación con los niveles de los individuos entrevistados, en donde se aprecia que se mantiene la tendencia que se venía observando desde 1990: un alto grado de homogamia para las personas con mayores niveles educacionales y menor para los niveles de poca escolaridad; con una tendencia a la baja del primer grupo y al alza del segundo.

${ }^{28}$ En concreto, se aleatorizaron, sin reemplazo, los niveles educacionales entre los padres.

${ }^{29} \mathrm{Si}$ se calculara nuevamente el emparejamiento selectivo para los resultados de la tabla 8 se obtendría una matriz llena de 1 . 
de se puede apreciar que los porcentajes totales o marginales coinciden con los de la tabla 6 , sólo cambia la distribución entre cada una de las clasificaciones de los niveles educacionales.

Tabla 8. DISTRIBUCIÓN POR NIVEL EDUCACIONAL DE LOS PADRES DE LOS ENCUESTADOS DEL ESCENARIO BENCHMARK

\begin{tabular}{|l|rrrrr|}
\hline Educ. madre & Sin educación & Básica & Media & Superior & Total \\
\hline Sin educación (\%) & 36,72 & 14,69 & 8,54 & 2,15 & 62,10 \\
Básica (\%) & 13,72 & 5,49 & 3,19 & 0,80 & 23,19 \\
Media (\%) & 7,39 & 2,96 & 1,72 & 0,43 & 12,50 \\
Superior (\%) & 1,30 & 0,52 & 0,30 & 0,08 & 2,20 \\
Total (\%) & 59,13 & 23,65 & 13,75 & 3,47 & 100,00 \\
\hline
\end{tabular}

Fuente: Elaboración del autor a partir de la encuesta Casen 2013.

El siguiente paso de la simulación era "aleatorizar a los padres de los individuos". En la práctica se aleatorizó el nivel educacional de éstos, y se optó por hacerlo con ambos progenitores, aunque de haberlo hecho sólo con uno de ellos los resultados no cambiaban sustancialmente. A partir de dicha aleatorización se obtuvo la distribución por clasificaciones $i j$ expuestas en la tabla 9 .

Como se aprecia, dado el gran tamaño muestral de la encuesta Casen, el proceso de aleatorización garantizó mínimas diferencias (recién al segundo decimal), entre la nueva distribución de niveles educacionales simulados y la distribución benchmark de la tabla 8 .

Tabla 9. DISTRIBUCIÓN, POST ALEATORIZACIÓN COMPUTACIONAL, DEL NIVEL EDUCACIONAL DE LOS "NUEVOS PADRES" DE LOS ENCUESTADOS.

\begin{tabular}{|l|rrrrr|}
\hline Educ. padre & Sin educación & Básica & Media & Superior & Total \\
\hline Sin educación (\%) & 36,69 & 14,68 & 8,59 & 2,15 & 62,10 \\
Básica (\%) & 13,69 & 5,52 & 3,17 & 0,82 & 23,20 \\
Media (\%) & 7,44 & 2,95 & 1,69 & 0,42 & 12,51 \\
Superior (\%) & 1,31 & 0,50 & 0,31 & 0,08 & 2,19 \\
Total (\%) & 59,13 & 23,65 & 13,75 & 3,47 & 100,00 \\
\hline
\end{tabular}

Fuente: Elaboración del autor a partir de la encuesta Casen 2013. 
En la etapa siguiente de la simulación se requería predecir un nuevo nivel educacional de los encuestados. Se optó por esto último $-\mathrm{y}$ no por calcular la escolaridad de éstos como variable continua-, pues con ello sólo se hubiera conocido la cantidad de años que hubiese estado estudiando el individuo, pero no si hubiera completado cierto nivel educacional en particular. Por ejemplo, si se predecía que el individuo tenía 14 años de escolaridad, ¿correspondería eso a educación superior de nivel técnico completo o educación superior profesional incompleta? Y dada la existencia del sheepskin effect ${ }^{30}$ (Ferrer y Riddell 2002; Hungerford y Solon 1987; Jaeger y Page 1996), se optó entonces por predecir, mediante un Probit Ordenado, el nivel educacional de las personas.

Para esto último, primero se utilizaron las mismas variables expuestas en la tabla 4, pero en vez de usar el nivel educacional de uno de los padres (que sólo permitía observar el efecto de la movilidad educacional intergeneracional o MEI), se incluyeron nueve variables dicotómicas (diez al considerar la categoría base), donde cada una indicaba qué combinación original de nivel educacional poseían sus progenitores reales (sin simulaciones). ${ }^{31}$ Los efectos marginales de la predicción anterior, para la categoría educación superior, se presentan en la tabla A.3 en Anexos. ${ }^{32}$

Posteriormente, se predijo un nuevo nivel educacional para cada individuo ponderando los coeficientes estimados (obtenidos del Probit Ordenado) por su variable correspondiente incluida en dicho modelo, con la finalidad de obtener un valor predicho de la variable latente. ${ }^{33}$ Ésta, posteriormente, determinaría el nuevo nivel educacional del individuo,

${ }^{30}$ Este efecto hace referencia al papel que cumple la señal de concreción del grado académico en lo que respecta a los ingresos.

${ }^{31}$ Se tomaron los mismos cuatro niveles usados hasta el momento (sin educación, básica, media y superior), pero no hay 16 variables dicotómicas ya que, por ejemplo, se usa una misma variable para indicar que el padre posee básica y la madre media o para indicar que el padre posee media y la madre básica, y así con todas las demás celdas de la matriz de la tabla 7.

${ }^{32}$ En la tabla A.3 se incluyen dos columnas. En la primera se muestra la estimación sin agregar el set de 9 variables dicotómicas, mientras que en la segunda se ofrecen los resultados incorporándolas.

${ }^{33}$ Que corresponde a la propensión del individuo a educarse. 
dependiendo de entre qué cortes haya quedado posicionada. ${ }^{34}$ Es importante mencionar que para este caso se construyeron nuevas variables dicotómicas que incluían la nueva combinación simulada/aleatorizada de niveles educacionales de los padres. Y son estas nuevas variables las que se ponderaron por los coeficientes correspondientes de las dicotómicas originales ${ }^{35}$ obteniéndose así el nuevo nivel educacional predicho de cada individuo para un escenario donde no hubiese habido homogamia.

Debe mencionarse que la forma en que fueron construidas las variables dicotómicas permitió recoger un efecto no sólo de la MEI, sino que también de emparejamiento selectivo, esto debido a que cada variable identificaba: i) el nivel educacional de los padres (lo que permite identificar el impacto de la MEI) y ii) su distribución de entre ambos progenitores (lo que hace posible distinguir el efecto del emparejamiento selectivo).

Finalmente, con esto último se predijeron nuevos ingresos laborales para cada persona, realizando ${ }^{36}$ una regresión de Mínimos Cuadrados Ordinarios (MCO) para los hombres y utilizando el método de Heckman $^{37}$ (1979) para las mujeres. Los regresores empleados son los indicados por Mincer (1974) — esto es, la escolaridad, la experiencia y la experiencia al cuadrado- - pero en vez de usar la escolaridad se ocuparon variables dicotómicas de los niveles educacionales; a su vez, se utilizó como variables explicativas el set de nueve dummies relativas al tipo de clasificación $i j$ del nivel educacional de los padres del individuo. Esto último se hizo por dos razones. La primera, para ver el efecto del emparejamiento selectivo en los ingresos laborales de los individuos, en donde era posible que existiesen efectos relativos a habilidades cognitivas y no cognitivas que fuesen otorgadas por los padres, así como redes de contacto u otras vías mediante las cuales el nivel educacional de los

34 Se habla de "cortes" cuando se hace referencia a los umbrales bajo los cuales el nivel de la variable latente debe ser evaluado, para finalmente clasificar el nivel educacional efectivo que tendrá el individuo. Todo esto a la luz de la metodología del Probit Ordenado.

${ }^{35}$ Para las demás variables se usan sus observaciones originales; no son simuladas.

36 Todas las estimaciones presentan errores estándar robustos a heterocedasticidad.

${ }^{37}$ Se utiliza el método de Heckman para corregir el sesgo de selección de la participación en el mercado laboral, usando como variable de exclusión el si la mujer estaba casada o no. 
padres afectase los ingresos laborales. Segundo, para identificar el efecto que, sobre los ingresos, tiene el nivel educacional del individuo sin el sesgo que podría provenir del efecto del nivel educacional de los padres también sobre los ingresos.

Luego se procedió a ponderar las variables de cada individuo por sus coeficientes correspondientes, pero usando los niveles educacionales simulados en vez de los observados, y con los mismos valores también simulados del set de nueve variables dicotómicas usado para predecir el nivel educacional. De esta forma, pudo estimarse un ingreso de la ocupación principal predicho. Las regresiones para hombres y mujeres se encuentran en la tabla A.4 de anexos.

A continuación, se complejizó el procedimiento para generar la nueva distribución de ingresos. Esto debido a que, si la simulación se hubiese llevado a cabo de forma directa, tal como se indicó recién, tanto la nueva distribución de niveles educacionales, como la del ingreso de la ocupación principal hubieran tenido una varianza considerablemente subestimada. Gran parte de este fenómeno sucedería porque se estaría prediciendo $^{38}$ un set limitado de variables de resultado, dado que la combinatoria de observaciones, producida por las diferentes variables explicativas utilizadas, también sería limitada. Es decir, se perdería la varianza que hubiesen generado las variables no observables, la cual se asumiría como idéntica entre individuos con iguales variables observables. Y dado que el proceso de simulación contó con dos etapas (una que predijo un nivel de escolaridad y la segunda que, a partir de la primera, generó ingresos), entonces en la segunda no sólo se generaría el inconveniente ya expuesto, sino que se exacerbaría aún más, dado que las variables explicativas de los niveles educacionales (que son un insumo para la segunda predicción) también tendrían una menor varianza, producto de su predicción en la primera etapa.

Este proceder permitiría sólo identificar el Gini entre las diferentes categorías $^{39}$ observadas y no al interior de éstas, porque este indicador sería nulo en el último caso, por depender de la construcción metodológica. Para lidiar con esta dificultad, este trabajo empleó dos meto-

${ }^{38}$ Nótese que la predicción se haría a partir de los coeficientes estimados (efectos promedio). Por lo cual es claro que la varianza de la variable de resultado a estimar será menor que la real.

${ }^{39}$ Que surgen a partir de las variables explicativas utilizadas. 
dologías diferentes dirigidas a estimar el Gini "real" de los ingresos simulados.

La primera de ellas propone aplicar el mismo procedimiento de la simulación a las variables originales (no a las simuladas). Esto es, se trata de que al predecir el nivel educacional habría que estimarlo con los valores observados ${ }^{40}$ del set de nueve variables dicotómicas, respectivas a las clasificaciones de emparejamiento de los padres de los individuos, y no con los valores simulados. Luego, con ese nivel educacional predicho, se estimarían los ingresos, para finalmente aplicar el cálculo del Gini sobre dicha distribución. En suma, sería como una especie de tratamiento placebo ${ }^{41}$ sobre los datos. ${ }^{42}$

La idea de lo anterior era que, si se cumplía, como supuesto, que no hubiese diferencias en el Gini intra categorías de la distribución simulada y la generada por el tratamiento placebo, entonces la diferencia entre ambos Gini subestimados debiese equivaler al efecto real del emparejamiento aleatorio en la desigualdad.

Paralelamente también se llevó a cabo otro procedimiento, posiblemente menos restrictivo que el anterior, el cual, por lo mismo, podría considerarse como más válido. Éste consistió en hacerse cargo de la variabilidad no considerada, radicada en las variables no observables. ${ }^{43}$ En un escenario ideal, si se conociese la distribución de los errores (y su varianza, puesto que la media ya es conocida porque se asume que es 0), se le podría sumar un error aleatorio, según su distribución, a cada individuo, sobre su variable predicha. Pero dado que no se conocen su

${ }^{40} \mathrm{O}$ sea, los efectivamente reportados en la encuesta.

${ }^{41}$ El placebo se ocupa en este caso para generar un escenario de control con el cual comparar el del tratamiento descrito.

42 Esto equivale a ver qué ocurriría para el "individuo promedio". Sin embargo, por semántica, se prefiere hablar de procedimiento placebo, ya que conceptualmente no tiene conexión analizar lo que ocurre con el individuo promedio en comparación con la distribución simulada. Es decir, si se están analizando desigualdades, no puede hablarse de la desigualdad de una sola persona (el individuo promedio).

${ }^{43}$ Como fue señalado anteriormente, en el caso de que la simulación se realizara de forma directa, surgía el problema de que las variables dependientes predichas poseían poca varianza, ya que en el análisis lograba incorporarse únicamente la información provista por las variables observables. Es por eso que, para hacernos cargo de las no observables, el tratamiento ideal sería sumarle un error aleatorio (siguiendo su distribución) a cada individuo, sobre su variable predicha. 
distribución (la de los errores) ni su varianza, se optó entonces por calcular los residuos de cada individuo, para posteriormente aleatorizarlos entre éstos.

Con respecto a este último proceso, se precisan algunas aclaraciones. Fue hecho para ambas etapas, la que estimaba los niveles educacionales y la que lo hacía con el ingreso de la ocupación principal, ya que en ambas se perdía variabilidad en las variables al no tomar en cuenta las no observables. Los residuos fueron calculados como la diferencia entre la variable dependiente observada y la variable predicha, estimada a partir de los valores reales de las variables explicativas, ${ }^{44}$ es decir, no el set simulado de variables dicotómicas de emparejamiento selectivo, ni del nivel educacional simulado (en el caso de la etapa de ingresos). Por otro lado, al momento de la aleatorización de los residuos entre los individuos, es razonable pensar que dicho proceso debiese haberse efectuado con reemplazo, es decir, el residuo de un individuo pudiese tocarle nuevamente a otro, lo cual es más razonable que restringir el set de posibles residuos a los individuos sucesivos. No obstante, se presentarán los resultados de simulaciones con y sin reemplazo, con el fin de mostrar la robustez de los resultados. También es importante aclarar que los residuos de la ecuación de ingresos fueron aleatorizados distinguiendo por sexo y nivel educacional, ante la posibilidad de que existiese heterocedasticidad.

Para minimizar el riesgo de que en el proceso de aleatorización de los residuos tocase una asignación muy favorable o desfavorable, en términos de desigualdad y de los otros resultados relevantes, se optó por simular este proceso 500 veces, de tal forma de poder generar intervalos de confianza para cada uno de dichos resultados.

Es importante mencionar que la predicción fue realizada con respecto al ingreso de la ocupación principal, y no a otro, debido a que éste era el que podía predecirse con mayor bondad de ajuste, usando en ello las variables de niveles educacionales. Esto era importante para poder

${ }^{44}$ La razón de ello es porque el vector de coeficientes estimados para las variables, que permite predecir posteriormente las variables dependientes, es calculado usando las observaciones originales (no simuladas), dado el conjunto de variables observables incluidas (y, por lo tanto, dado el de no observables excluidas); por ende, para poder recuperar la variabilidad perdida del modelo, producto de las no observables, es más apropiado usar los residuos con las variables originales, que permitieron calcular el vector de coeficientes antes mencionado. 
reducir al mínimo el problema de identificación del efecto del escenario contrafactual. Por otra parte, considerando que el nivel educacional también podía tener efectos en la determinación de otros ingresos no laborales, entonces se optó por calcular el Gini solamente sobre el ingreso de la ocupación principal, pues el efecto producido por emparejamiento selectivo se podría apreciar más directamente, reduciendo otros potenciales sesgos. ${ }^{45}$

Finalmente, se debe aclarar que los resultados relativos a los ingresos de la ocupación principal predichos, presentados en la siguiente sección, fueron ajustados, de tal forma que sólo se tomó en cuenta a aquellos individuos que originalmente reportaron una cifra de sus ingresos de su ocupación principal. Es decir, no se consideró a todos quienes se les predijo algún valor para esta variable pero que originalmente no lo tenían.

\subsection{Datos}

La simulación realizada fue llevada a cabo empleando los datos de la encuesta Casen 2013. Ésta está a cargo del Ministerio de Desarrollo Social, emplea un muestreo por clusters y entrevistas cara a cara, y otorga representatividad a nivel nacional. La muestra incluye a 218.491 personas de todas las regiones del país. Además, este instrumento provee información detallada sobre los ingresos de hogares e individuos, en conjunto con un amplio set de preguntas relativas a una completa caracterización socioeconómica de las personas, incluyendo algunas relacionadas con la educación de los padres de los encuestados.

Todo lo anterior permitió llevar a cabo la simulación mencionada, pues fue posible estimar los ingresos, niveles educacionales e índices de desigualdad. Por lo demás, los anteriores indicadores de MEI y empare-

${ }^{45}$ Esto, porque al estar sesgado el nivel educacional predicho, cada vez que se usase una variable en estas condiciones para explicar otra, esta última también lo estaría. Por lo tanto, mientras menos variables (del tipo de ingresos) se intenten explicar con el nivel educacional, menos se sesgará la variable final observada (en el caso de los ingresos, la variable observada puede ser, por ejemplo, el ingreso autónomo, el cual se compone de otras variables, como los ingresos laborales y otros ingresos autogenerados, los cuales, considerados cada uno de ellos, podrían estar sesgados si es que fuesen explicados por el nivel educacional también). 
jamiento selectivo, al igual que la distribución benchmark o de referencia, también fueron calculados utilizando la encuesta Casen. ${ }^{46}$

\subsection{Resultados 47}

Debe mencionarse que estos resultados representan un equilibrio parcial, por lo que eventualmente pudieran existir otras consecuencias de un escenario como el aquí descrito y que tal vez pudiesen afectar de modo indirecto a dichos resultados.

Las tablas 10 y 11 muestran los principales hallazgos de todas las simulaciones ya descritas. En la primera se presentan los relativos al caso con imputaciones y en la segunda se exponen las estimaciones hechas sobre la submuestra que incluye sólo a los individuos que reportaron el nivel educacional de ambos padres. Las columnas referentes a la metodología de suma de residuos (tanto con y sin reemplazo) pueden compararse con la primera columna, donde se exhiben los datos de la muestra total y los de la submuestra, respectivamente; mientras que las de la estimación directa deben compararse con la columna de la falsa estimación o placebo, como se ha llamado. Cabe destacar que, dado que para la "simulación de suma de residuos" se realizó 500 veces el proceso de aleatorización, los resultados se presentan en intervalos de confianza (al 95 por ciento).

Si bien se podrían considerar los resultados de la columna de la estimación de suma de residuos con reemplazo de la tabla 10 (con imputaciones) como los resultados de mayor validez, se presentan también los demás a fin de mostrar la robustez de los resultados. En efecto, a modo global, se pueden extraer los siguientes resultados a partir de cómo serían las distribuciones de ingresos y de nivel educacional de la sociedad chilena, si los padres de la generación actual no hubiesen presentado un emparejamiento selectivo en escolaridad:

${ }^{46}$ Las mediciones de MEI y emparejamiento selectivo presentadas en la sección 2 de "Contexto", y que corresponden a años distintos a 2013, fueron calculadas también con la encuesta Casen de los correspondientes lustros. Tal como se indica en la fuente de los gráficos y tablas.

${ }^{47}$ Los ingresos utilizados para las estimaciones de este trabajo corresponden a los de la metodología tradicional de Casen, es decir, con ajuste por cuentas nacionales. 
1. En todas las especificaciones se experimenta una baja en la desigualdad, medida mediante el coeficiente de Gini, en donde se observa una caída de 5 puntos porcentuales, pasando de 0,48 a 0,43 . Para poner en perspectiva esta cifra, nótese que el Gini del ingreso de la ocupación principal en 1990 era de 0,53, es decir, esta disminución sería el equivalente a la reducción hecha por Chile durante casi un cuarto de siglo. En las demás simulaciones también se corrobora una caída en este índice, aunque menor.

2. Por otro lado, la desigualdad en los extremos de la distribución de ingresos, medida mediante el ratio 10/10 parece no haber disminuido. Es más, aumentó levemente, pasando de 6,3 a 6,4. Esta alza es leve, teniendo en cuenta que el nivel de este indicador en 1990 era de 7,7. Este resultado va en la misma dirección que en las demás simulaciones, a excepción del caso de la simulación directa para la submuestra de la tabla 11.

3. Quizás uno de los resultados más interesantes de este trabajo sea, además de la importante caída en desigualdad, que el ingreso laboral promedio (o de la ocupación principal) cae significativamente. Pasando de poco más de $\$ 500.000$ a poco más de $\$ 425.000$, monto que corresponde al ingreso promedio de la ocupación principal en el año 1998 en Chile. ${ }^{48}$ Esto equivale a una caída de 15 por ciento de los salarios. Las demás simulaciones también muestran una caída importante del ingreso promedio, en algunos casos mayor y en otros menor. Por otro lado, en todos los escenarios se aprecia una disminución de la desviación estándar de la distribución de ingresos laborales.

4. Por último, se observa que la distribución de niveles educacionales tiende a disminuir la densidad en los extremos y abultarse más en los niveles centrales. En efecto, se aprecia un efecto positivo, al reducirse la cantidad de individuos sin escolaridad o con educación básica incompleta ("Sin educación"), desde 31 por ciento a 26 por ciento. Sin embargo, ello viene asociado a una caída en una magnitud similar, desde 12 por ciento a 7 por ciento, en la cantidad de personas con educación superior, en donde de ese 10 por ciento (5 por ciento que se desplazó cada una) que pertenecía a dichas categorías pasa poco más de 3 por ciento a educación media y 7 por ciento a educación básica, tal que también, presumiblemente, exista un porcentaje que se haya transferido de media a básica. La tendencia de estos resultados se repite en las otras simulaciones.

\footnotetext{
${ }^{48}$ Ajustado por inflación.
} 
Tabla 10. RESULTADOS DE LAS DIVERSAS METODOLOGÍAS DE SIMULACIÓN, APLICADAS A LA MUESTRA CON IMPUTACIONES.

\begin{tabular}{|c|c|c|c|c|c|c|}
\hline & & $\begin{array}{c}\text { Valores } \\
\text { originales } \\
\text { muestra }\end{array}$ & $\begin{array}{l}\text { Simulación } \\
\text { de iteración } \\
\text { con suma de } \\
\text { residuos con } \\
\text { reemplazo }\end{array}$ & $\begin{array}{l}\text { Simulación } \\
\text { de iteración } \\
\text { con suma de } \\
\text { residuos sin } \\
\text { reemplazo }\end{array}$ & $\begin{array}{c}\text { Simulación } \\
\text { placebo }\end{array}$ & $\begin{array}{l}\text { Simulación } \\
\text { directa }\end{array}$ \\
\hline \multirow{6}{*}{$\begin{array}{l}\text { Ingreso } \\
\text { de la } \\
\text { ocu- } \\
\text { pación } \\
\text { principal }\end{array}$} & Gini & 0,48 & {$[0,426 ; 0,428]$} & {$[0,440 ; 0,441]$} & 0,24 & 0,22 \\
\hline & Ratio 10/10 & 6,3 & {$[6,40 ; 6,44]$} & {$[6,70 ; 6,72]$} & 2,68 & 2,80 \\
\hline & Promedio & 501.020 & $\begin{array}{r}\text { [426.583; } \\
428.284]\end{array}$ & $\begin{array}{r}\text { [452.695; } \\
453.524]\end{array}$ & 303.264 & 281.209 \\
\hline & $\begin{array}{l}\text { Desviación } \\
\text { estándar }\end{array}$ & 775.985 & $\begin{array}{l}{[547.318} \\
564.487]\end{array}$ & $\begin{array}{r}\text { [622.280; } \\
\text { 633.050] }\end{array}$ & 139.639 & 122.893 \\
\hline & Mínimo & 2.270 & [2.972; 3.209] & {$[1.777 ; 1.881]$} & 38.208 & 38.208 \\
\hline & Máximo & 43.500 .000 & $\begin{array}{l}\text { [22.358.910; } \\
\text { 23.956.604] }\end{array}$ & $\begin{array}{r}\text { [42.055.734; } \\
44.714 .862]\end{array}$ & 1.274 .917 & 1.275 .243 \\
\hline \multirow{8}{*}{$\begin{array}{l}\text { Nivel } \\
\text { educa- } \\
\text { cional }\end{array}$} & $\begin{array}{l}\text { Sin educación } \\
\text { (Frec.) }\end{array}$ & 5.417 .480 & $\begin{array}{r}\text { [4.498.602; } \\
4.502 .580]\end{array}$ & $\begin{array}{l}\text { [4.476.164; } \\
4.479 .683]\end{array}$ & 4.717.166 & 3.722 .415 \\
\hline & $\begin{array}{l}\text { Sin educación } \\
(\%)\end{array}$ & 31,49 & {$[26,15 ; 26,17]$} & {$[25,91 ; 25,93]$} & 27,31 & 21,55 \\
\hline & $\begin{array}{l}\text { Educación } \\
\text { básica (Frec.) }\end{array}$ & 4.198 .088 & $\begin{array}{r}{[5.496 .514 ;} \\
5.502 .014]\end{array}$ & $\begin{array}{r}\text { [5.475.268; } \\
\text { 5.480.247] }\end{array}$ & 5.360 .432 & 6.693.344 \\
\hline & $\begin{array}{l}\text { Educación } \\
\text { básica (\%) }\end{array}$ & 24,40 & {$[31,95 ; 31,98]$} & {$[31,70 ; 31,73]$} & 31,03 & 38,75 \\
\hline & $\begin{array}{l}\text { Educación } \\
\text { media (Frec.) }\end{array}$ & 5.463 .920 & $\begin{array}{r}\text { [5.985.929; } \\
5.990 .752]\end{array}$ & $\begin{array}{r}\text { [5.993.282; } \\
5.997 .880]\end{array}$ & 6.322.098 & 6.595 .471 \\
\hline & $\begin{array}{l}\text { Educación } \\
\text { media (\%) }\end{array}$ & 31,76 & {$[34,79 ; 34,82]$} & {$[34,70 ; 34,72]$} & 36,60 & 38,18 \\
\hline & $\begin{array}{l}\text { Educación su- } \\
\text { perior (Frec.) }\end{array}$ & 2.125 .105 & $\begin{array}{l}{[1.214 .901 ;} \\
1.217 .894]\end{array}$ & $\begin{array}{r}\text { [1.320.408; } \\
1.323 .301]\end{array}$ & 873.421 & 261.887 \\
\hline & $\begin{array}{l}\text { Educación } \\
\text { superior (\%) }\end{array}$ & 12,35 & {$[7,06 ; 7,08]$} & {$[7,64 ; 7,66]$} & 5,06 & 1,52 \\
\hline
\end{tabular}

Fuente: Elaboración del autor a partir de la encuesta Casen 2013. 
Tabla 11. RESULTADOS DE LAS DIVERSAS METODOLOGÍAS DE SIMULACIÓN, APLICADAS A LA MUESTRA SIN IMPUTACIONES

\begin{tabular}{|c|c|c|c|c|c|c|}
\hline & & $\begin{array}{l}\text { Muestra } \\
\text { restringida }\end{array}$ & $\begin{array}{c}\text { Simulación } \\
\text { de iteración } \\
\text { con suma de } \\
\text { residuos con } \\
\text { reemplazo }\end{array}$ & $\begin{array}{l}\text { Simulación } \\
\text { de iteración } \\
\text { con suma de } \\
\text { residuos sin } \\
\text { reemplazo }\end{array}$ & $\begin{array}{c}\text { Simulación } \\
\text { placebo }\end{array}$ & $\begin{array}{c}\text { Simulación } \\
\text { directa }\end{array}$ \\
\hline \multirow{6}{*}{$\begin{array}{c}\text { Ingreso } \\
\text { de la ocu- } \\
\text { pación } \\
\text { principal }\end{array}$} & Gini & 0,51 & $\begin{array}{r}{[0,462 ;} \\
0,463]\end{array}$ & {$[0,463 ; 0,464]$} & 0,27 & 0,26 \\
\hline & Ratio $10 / 10$ & 7,3 & {$[7,56 ; 7,60]$} & {$[7,596 ; 7,627]$} & 3,04 & 2,96 \\
\hline & Promedio & 592.710 & $\begin{array}{l}\text { [494.775; } \\
\text { 496.548] }\end{array}$ & $\begin{array}{r}\text { [506.019; } \\
507.159]\end{array}$ & 333.165 & 311.297 \\
\hline & $\begin{array}{l}\text { Desv. } \\
\text { estándar }\end{array}$ & 935.981 & $\begin{array}{r}\text { [699.797; } \\
719.797]\end{array}$ & $\begin{array}{l}\text { [745.513; } \\
765.538]\end{array}$ & 172.926 & 170.571 \\
\hline & Mínimo & 2.270 & $\begin{array}{l}\text { [3.319; } \\
3.494]\end{array}$ & [3.199; 3.312] & 41.140 & 41.140 \\
\hline & Máximo & 43.500 .000 & $\begin{array}{r}\text { [32.557.526; } \\
34.969 .131]\end{array}$ & $\begin{array}{r}{[44.925 .607} \\
47.577 .842]\end{array}$ & 1.400 .202 & 1.648 .179 \\
\hline \multirow{8}{*}{$\begin{array}{l}\text { Nivel } \\
\text { educa- } \\
\text { cional }\end{array}$} & $\begin{array}{l}\text { Sin educa- } \\
\text { ción (Frec.) }\end{array}$ & 839.650 & $\begin{array}{l}\text { [612.414; } \\
614.338]\end{array}$ & $\begin{array}{r}613.969 ; \\
615.881]\end{array}$ & 264.036 & 127.471 \\
\hline & $\begin{array}{l}\text { Sin educa- } \\
\text { ción (\%) }\end{array}$ & 15,21 & {$[11,10 ; 11,13]$} & {$[11,09 ; 11,12]$} & 4,77 & 2,30 \\
\hline & $\begin{array}{l}\text { Educación } \\
\text { básica } \\
\text { (Frec.) }\end{array}$ & 1.395 .572 & $\begin{array}{c}\text { [1.702.050; } \\
1.705 .366]\end{array}$ & $\begin{array}{c}\text { [1.704.889; } \\
1.708 .055]\end{array}$ & 2.015 .886 & 1.903 .782 \\
\hline & $\begin{array}{l}\text { Educación } \\
\text { básica (\%) }\end{array}$ & 25,29 & $\begin{array}{r}{[30,84 ;} \\
30,90]\end{array}$ & {$[30,78 ; 30,84]$} & 36,40 & 34,38 \\
\hline & $\begin{array}{l}\text { Educación } \\
\text { media } \\
\text { (Frec.) }\end{array}$ & 2.093.977 & $\begin{array}{l}\text { [2.382.725; } \\
2.386 .088]\end{array}$ & $\begin{array}{c}\text { [2.377.964; } \\
2.381 .521]\end{array}$ & 2.728 .559 & 3.341 .099 \\
\hline & $\begin{array}{l}\text { Educación } \\
\text { media (\%) }\end{array}$ & 37,94 & $\begin{array}{l}{[43,17 ;} \\
43,24]\end{array}$ & {$[42,94 ; 43,00]$} & 49,27 & 60,33 \\
\hline & $\begin{array}{l}\text { Educación } \\
\text { superior } \\
\text { (Frec.) }\end{array}$ & 1.189 .654 & $\begin{array}{l}\text { [816.183; } \\
\text { 818.542] }\end{array}$ & $\begin{array}{r}\text { [835.854; } \\
838.134]\end{array}$ & 529.653 & 165.782 \\
\hline & $\begin{array}{l}\text { Educación } \\
\text { superior } \\
\text { (\%) }\end{array}$ & 21,56 & {$[14,79 ; 14,83]$} & {$[15,09 ; 15,13]$} & 9,56 & 2,99 \\
\hline
\end{tabular}

Fuente: Elaboración del autor en base a encuesta Casen 2013. 


\section{INTERPRETACIONES}

\subsection{Análisis}

Podría rechazarse la hipótesis antes planteada según la cual el nivel educacional de los padres aportaría de manera creciente, pero a tasas marcadamente decrecientes, en la propensión de escolarización de sus hijos, puesto que, luego de la simulación, se observa una caída importante en la cantidad de individuos con educación superior. Ello quiere decir que, en muchos casos, no basta con tener un solo padre con alta educación para que el hijo también la tenga, sino que se necesita del aporte que hacen ambos progenitores en su nivel educacional. Es decir, en el agregado, no aumenta la cantidad de personas con educación superior, incremento que hubiese ocurrido si bastase sólo con el aporte del padre más educado. En la simulación, al disminuir el emparejamiento selectivo, aumentó la cantidad de personas con al menos un padre más educado, por ende, de ser cierto que el aporte en la educación del individuo, por parte del segundo progenitor, no fuese marginalmente importante, entonces se debiese haber observado un incremento en el porcentaje de personas con mayores niveles educacionales. Puesto que ello no fue así, por contradicción, la hipótesis puede rechazarse. Nótese, eso sí, que podría pensarse que el aumento fue absorbido por gente con educación media, pero el porcentaje de incremento de este nivel fue menor que la reducción de quienes tenían educación superior.

En otro ámbito, nótese que las simulaciones hechas de manera directa, comparadas con la falsa simulación, muestran diferencias más acotadas en los resultados que los predichos mediante la metodología de sumar los residuos. Ello puede deberse a que no se haya cumplido el supuesto identificador de que no hubiese diferencias entre el Gini intra categorías de la distribución simulada y el Gini intra categorías de la distribución generada por el tratamiento placebo. Por lo tanto, es probable que sólo se esté observando la diferencia de los Gini entre categorías, lo que explicaría que éstas sean más acotadas que las presentadas por el otro procedimiento. De todas formas, constituyen un activo valioso para verificar la robustez de las estimaciones.

En relación con los cuatro puntos analizados en el apartado anterior, se infiere que: 
1. Si bien es una cuantía considerable la caída en 5 puntos del Gini que se presenta, ésta podría ser mucho mayor para las próximas generaciones, en el caso de que las parejas de hoy no presentasen emparejamiento selectivo. Esto ocurriría debido a que, en el análisis presentado, casi 8 de cada 10 personas poseían originalmente ambos padres sin educación o con educación básica. De esta forma, el impacto de aleatorizar a los padres no sería muy alto, ya que, tal como lo muestra la distribución benchmark, en dicho escenario seguirían quedando poco más de 7 de cada 10 individuos tanto con padre o madre con educación básica o sin educación. Esto quiere decir que la redistribución de padres educados con educación media o superior no tendría un efecto tan significativo en las variables de resultado, ya que constituían un bajo porcentaje del total. Por lo tanto, si se analiza con esta perspectiva, entonces efectivamente puede decirse que los resultados aquí expuestos son de gran impacto, puesto que bastó redistribuir la poca gente más educada que había para generar alteraciones importantes en la desigualdad del país.

Si además se observa la tabla A.4 (ver Anexos) puede concluirse que los ingresos laborales también se ven afectados de forma directa por la redistribución de padres, en algunos casos positivamente y en otros de manera negativa. Por ejemplo, se ve que, si se comparan dos parejas de padres, una en la que ambos progenitores tienen educación básica y otra con ambos sin educación, entonces la hija ${ }^{49}$ de los primeros tendría en promedio 9 por ciento $(0,086)$ más de salario que la hija de los segundos. Esto es menor de lo que tendrían ambas hijas (sumado) en el caso de que cada una tuviese un padre con educación básica y el otro sin educación. En ese caso, entre ambas tendrían 13 por ciento $(0,064+0,064=0,128)$ más que la hija de la pareja con ambos padres sin educación del primer escenario. En este caso entonces sí se observa un efecto positivo ${ }^{50}$ en salarios de una situación sin emparejamiento selectivo.

No obstante, el ejemplo anterior contrasta con un escenario análogo pero referido a una pareja con educación superior y la otra sin educación, en cuyo caso el primer porcentaje (con emparejamiento

49 "La hija" viene a representar a cualquiera de los individuos a los cuales se les está prediciendo el ingreso mediante las estimaciones de la tabla A.4.

${ }^{50}$ Pues, lógicamente, el 13 por ciento del segundo caso es mayor que el 9 por ciento del primero. 
selectivo) ascendería a 51 por ciento y el segundo (sin emparejamiento selectivo) llegaría sólo a 26 por ciento. Aquí, al eliminar la homogamia, se evidenciaría un impacto negativo en los salarios.

2. Es interesante notar que la caída en desigualdad que se observa en el Gini aparentemente proviene de una contracción de la distribución de ingresos de los tramos medios, dado que la desigualdad de los deciles extremos, de acuerdo al ratio 10/10, parece mantenerse similar.

3. Teniendo en cuenta que los individuos con educación superior perciben salarios considerablemente mayores que los del resto de los niveles educacionales (Beyer 2011), la caída en el porcentaje de personas con dicho nivel implica un impacto negativo mucho mayor en términos de los salarios promedio, que el impacto positivo producido por la menor proporción de gente sin educación.

4. Si bien no es trivial llegar a una conclusión respecto a si la nueva distribución de niveles educacionales es mejor o peor que la original, sí se puede decir que es positiva la disminución de gente sin educación, aunque cae también el porcentaje de individuos de mayor calificación. Por otra parte, este escenario es más virtuoso si se trata de otorgar oportunidades con mayor equidad, algo que a largo plazo podría ser más beneficioso; porque, el nuevo emparejamiento selectivo, hipotéticamente, ocurriría bajo un piso educacional más elevado.

De todas formas, es interesante observar detalladamente la tabla A.3 (ver Anexos). En ella se muestran los efectos marginales de las variables explicativas del Probit Ordenado, a fin de estimar el nivel educacional para la categoría de educación superior. Como se vio, si bien la proporción de personas con este nivel cae en la simulación, se puede observar que en un escenario con bajo emparejamiento selectivo (en contraste a otro con alto emparejamiento selectivo), mediante la mayoría de las distintas clasificaciones $i j$, mejoraría la propensión de tener educación superior. Sin embargo, seguramente, el aumento de esta variable (la de propensión) no sería suficiente para superar el corte (del Probit Ordenado) que clasificaría al individuo como alguien con este nivel. Debe considerarse además que la excepción a este análisis se da cuando se pasa de un escenario con homogamia elevada a otro de baja homogamia para las clasificaciones de educación superior y sin educación. Es decir, la propensión no mejora más, en el agregado, al generar más parejas de un padre con educación superior 
y el otro sin educación. Para este caso, es mejor en el total que existan parejas sólo de personas con educación superior y otras sólo de individuos sin educación.

Para ilustrar con claridad lo anterior, revísese un ejemplo: si se observan los efectos marginales de la tabla A.3 y se toma a un individuo con ambos padres de máximo nivel educacional y a otro individuo de ambos padres con el mínimo, se vería que el primero tendría 67 por ciento más de probabilidades de tener educación superior que el segundo. Si se supone, en cambio, como se hizo en la simulación, que los mismos individuos tienen un padre o madre con educación superior y su otro progenitor sin educación, en ese caso ambos tendrían 28 por ciento más de probabilidades de tener educación superior que alguien cuyos dos padres no poseen educación. Es decir, en el agregado, el haber distribuido a los padres de estos dos individuos termina generando una propensión de 56 por ciento ${ }^{51}$ a que alguien obtenga educación superior. Esto contrasta con la realidad de emparejamiento selectivo vigente, en el cual la probabilidad de que alguien tuviese educación superior, en el agregado, sería 11 por ciento mayor. Ahora, si se realizara el mismo ejercicio con la mayoría de las demás clasificaciones $i j$ se vería que ahí el caso es distinto, y que en un escenario sin homogamia en el agregado sí mejoraría la propensión de tener educación superior. Sin embargo, y como ya se ilustró anteriormente, la proporción de padres sin educación es tan elevada que al final ello termina "nivelando hacia abajo".

Por último, es importante añadir que, a futuro, si la oferta laboral femenina continúa expandiéndose como lo ha hecho hasta ahora (Beyer 2011), es probable que el alcance del impacto del emparejamiento selectivo quede de manifiesto con mayor fuerza. Debido a que si bien hoy existe un emparejamiento selectivo elevado, que aumenta la desigualdad, su impacto no alcanza su máximo potencial, ya que al haber un porcentaje importante de mujeres que no trabaja el emparejamiento selectivo en esos casos aún no se traduce en diferencia de ingresos.

${ }^{51}$ Cifra que proviene de sumar toscamente el 28 por ciento de ambos individuos. Evidentemente, en una economía con dos individuos como la descrita en el ejemplo, dicha probabilidad es incluso menor, ya que sería la unión de ocurrencia de dos eventos, por lo que habría que aún restarle la intersección de ocurrencia de ambos. 


\subsection{Implicancias para políticas públicas}

Tal como señala Torche (2010), un elevado nivel de segregación se traduce en elevados índices de homogamia, ya que la primera reduce las probabilidades de encontrar una pareja de distinto nivel socioeconómico. Este trabajo, por su parte, permite pensar en una hipótesis complementaria a la anterior. Si a dicho contexto se le suma una baja movilidad educacional intergeneracional, entonces los hijos que surgiesen de tales familias también heredarán el nivel educacional de sus padres. Ahora, si estos hijos viven en un entorno segregado, desde el establecimiento educacional al que asisten hasta en el barrio en donde habitan, frecuentarán otros hijos de un nivel socioeconómico similar. Esto, a su vez, sesgaría considerablemente sus probabilidades de emparejarse con alguien de otro nivel educacional, reforzando el ciclo de segregación.

El análisis expuesto es especialmente trascendente al verificarse la elevada magnitud y significancia estadística que tiene el set de variables de clasificaciones de los niveles educacionales de los padres que se presentan en la tabla A. $3^{52}$ (en Anexos).

Lo anterior ilustra cómo, bajo un contexto de poca movilidad educacional intergeneracional, el emparejamiento selectivo es tanto consecuencia y causa de la segregación social. Por lo tanto, asumiendo constante la movilidad educacional, una manera de terminar con este círculo vicioso que menoscaba la meritocracia es realizar políticas públicas que apunten a reducir la segregación. Por ejemplo, para el contexto chileno, medidas como el fin al financiamiento compartido en los colegios subvencionados o políticas urbanísticas ${ }^{53}$ que inhiban la segregación podrían permitir romper con este ciclo.

Con respecto al primer punto, puede mencionarse que en una simulación realizada por Elacqua y Santos (2013) ellos muestran que si los estudiantes asistieran al establecimiento educacional más cercano con respecto a sus hogares (escenario contrafactual), la segregación - medida usando diversos índices desarrollados en la literatura - sería menor de lo que es en la realidad. Los autores esgrimen que la existen-

52 Si bien en ésta se exhiben sólo los efectos marginales para la categoría de educación superior, los resultados de las demás también son altamente significativos y de gran magnitud.

${ }^{53}$ En especial en ciudades fuera de la Región Metropolitana, donde la segregación aún no está totalmente arraigada y queda más espacio de acción. 
cia de barreras que establecen las escuelas (como precios o requisitos de admisión) incrementan la segregación escolar por sobre el efecto que tiene la segregación residencial. De esta forma, reducir o eliminar dichas barreras permitiría atacar este problema.

Por otra parte, con respecto a las políticas urbanas que se podrían implementar, es interesante revisar las propuestas sugeridas por Brain et al. (2007), dentro de las cuales figuran tres posibles líneas de una política de suelo para la integración social: i) aumentar la disponibilidad de suelo bien localizado y urbanizado para vivienda social, ii) fomentar en los barrios populares la "gentrificación" sin expulsión ${ }^{54}$ e iii) incentivar el mercado de la vivienda usada. Todos estos factores apuntan a generar una mayor integración social que puede propiciar mayores espacios de encuentro entre personas de distintos niveles educacionales.

Siguiendo el trabajo de Frankel (2014) y Chade y Ventura (2002), también existiría la posibilidad de establecer esquemas impositivos que fomenten o desalienten, según se prefiera, el emparejamiento selectivo. En el trabajo de Chade y Ventura, por ejemplo, se describe el caso de Estados Unidos, en donde se aplican diferentes tasas de impuestos a los ingresos de las personas dependiendo de si estén casadas o estén solteras. En particular, en ese documento se muestra que, dependiendo del régimen impositivo, pueden establecerse incentivos de tal forma que se incrementen las preferencias por buscar parejas cuyos ingresos sean lo más distintos posible del propio, ya que en dicho caso la pareja podría gozar, en el total, de una deducción impositiva. No así en caso de que ambas personas estuviesen solteras o si la pareja ganase una cantidad similar de ingresos, en cuyo evento podrían terminar pagando más al estar casados. De esta manera, muestran los autores, la correlación de ingresos disminuiría.

En concreto, Chade y Ventura señalan que, si existiesen beneficios tributarios para los matrimonios, entonces el conjunto de potenciales parejas para cada individuo aumenta, disminuyendo la correlación de ingresos. De esta forma, conforme disminuyan los beneficios tributarios para los matrimonios, se reducen también los incentivos de buscar pare-

${ }^{54}$ La "gentrificación" alude a la renovación y revitalización de barrios por el proceso a través del cual hogares de clase media alta reemplazan a hogares de menores ingresos, aumentando los valores de las propiedades, alterando el ambiente construido y emergiendo un nuevo estilo de vida urbana. 
jas que posean mayores diferencias salariales, haciendo las decisiones maritales más selectivas, disminuyendo el número de matrimonios totales y aumentando la correlación de los ingresos de las parejas.

Como ya se ha mencionado, existen buenos argumentos a favor de reducir la homofilia y hasta aquí se han presentado algunos mecanismos disponibles. No obstante, esta investigación también ha mostrado evidencia en favor del emparejamiento selectivo (y con ello de la segregación), al menos para el contexto aquí analizado (con una generación de padres con alto porcentaje sin educación y bajo un escenario de altos retornos a la educación superior), en el que se aprecian mayores ingresos promedio y una mayor cantidad de personas con educación superior.

Es importante señalar que si el premio a la educación superior fuese menor que el que posee Chile en la actualidad, es posible que la disminución de personas con dicho nivel, producto de un escenario sin homogamia, tendría un efecto menor en los salarios. O que, dado los efectos marginales de la tabla A.3, si hubiese menos padres sin educación (como eventualmente ocurrirá para la próxima generación), entonces incluso podría aumentar la cantidad de individuos con educación superior, como se revisó en el apartado anterior.

Otra conclusión relevante a tener en cuenta es la referente a la heterogeneidad de efectos que existen entre las distintas variables de clasificaciones de niveles educacionales de los padres, sobre los ingresos y la propensión a escolarizarse de los individuos. En el último caso, dicho efecto puede provenir del capital cultural que proveen los padres; mientras que, en el primer caso, puede, por ejemplo, tener relación con las redes de contacto que éstos facilitan. Lo trascendental es que en algunos casos se ve que existe algo similar a un efecto par, en donde el padre con mayor educación logra aportar más favorablemente a la propensión a escolarizarse y al salario de su hijo; más, dígase, de lo que podría "perjudicarlo" una pareja de menor educación. Por otra parte, en otras de las clasificaciones $i j$ podría haber algo similar a un efecto "manzana podrida", y ocurriría exactamente lo contrario que en el caso anterior.

En suma, se espera que este trabajo sirva como insumo para la toma de decisiones políticas y la discusión de algunos de los costos y beneficios asociados a medidas que afecten directa o indirectamente el emparejamiento selectivo, la movilidad educacional intergeneracional o incluso la segregación. 


\section{CONCLUSIÓN}

Se presentó un análisis que se refiere a la existencia de factores estructurales de la sociedad chilena que limitan la distribución de oportunidades a los grupos de bajos ingresos. Ante ello surge como explicación plausible el hecho de que Chile posea elevados índices de homogamia o emparejamiento selectivo en escolaridad, lo que provoca dos efectos negativos en la desigualdad: uno directo, el cual implica que se formen familias de altos ingresos y otras de bajos ingresos y muy pocas de ingresos medios; y otro indirecto, de más largo plazo, referido a que una elevada homogamia, en conjunto con baja movilidad educacional intergeneracional pueden provocar resultados perjudiciales para la distribución de ingresos $-\mathrm{o}$ la desigualdad-, como fue presentado en la simulación del escenario contrafactual. Sin embargo, se constata que, de no haber existido un emparejamiento selectivo, disminuirían en 5 por ciento las personas con educación superior, producto de que tener al menos un padre de baja escolaridad parece ser más perjudicial que el aporte de tener al menos uno con alto nivel educacional. Lo anterior, además, repercute en una disminución de 15 por ciento de los salarios. En síntesis, se evidenciaría una caída en el Gini equivalente a lo que se ha avanzado en el último cuarto de siglo, junto a un escenario con menos gente sin educación y con más igualdad de oportunidades.

Si no se toman en cuenta estos factores estructurales que limitan considerablemente el actuar de otras políticas públicas que pudiesen atacar a la desigualdad de ingresos, todo indica que sólo algunos grupos privilegiados de la población contarán con los medios para poder surgir, y la alta educación y los altos ingresos se concentrarán sólo en ciertos grupos, provocándose así una trampa de desigualdad. 


\section{ANEXOS}

Tabla A.1. DISTRIBUCIÓN PORCENTUAL POR NIVEL EDUCACIONAL DE LA EDUCACIÓN DE LOS PADRES DE LOS ENCUESTADOS

\begin{tabular}{|c|c|c|c|c|c|}
\hline $\begin{array}{l}\text { Educ. padre } \\
\text { Educ. madre }\end{array}$ & Sin educación & Básica & Media & Superior & Total \\
\hline Sin educación (\%) & 44,22 & 6,95 & 2,20 & 0,28 & 53,65 \\
\hline Básica (\%) & 5,02 & 16,89 & 4,10 & 0,85 & 26,85 \\
\hline Media (\%) & 1,37 & 2,70 & 10,35 & 1,83 & 16,24 \\
\hline Superior (\%) & 0,09 & 0,31 & 0,78 & 2,07 & 3,25 \\
\hline Total (\%) & 50,70 & 26,85 & 17,43 & 5,03 & 100,00 \\
\hline
\end{tabular}

Fuente: Elaboración del autor en base a encuesta Casen 2013.

Tabla A.2. EMPAREJAMIENTO SELECTIVO DE LOS PADRES DE LOS ENCUESTADOS

\begin{tabular}{|l|cccc|}
\hline Educ. madre Educ. padre & Sin educación & Básica & Media & Superior \\
\hline Sin educación & 1,63 & 0,48 & 0,24 & 0,10 \\
Básica & 0,37 & 2,34 & 0,88 & 0,63 \\
Media & 0,17 & 0,62 & 3,65 & 2,24 \\
Superior & 0,05 & 0,35 & 1,38 & 12,68 \\
\hline
\end{tabular}

Fuente: Elaboración del autor en base a encuesta Casen 2013. 
Tabla A.3. EFECTOS MARGINALES DE PROBIT ORDENADO DEL NIVEL EDUCACIONAL CON VARIABLES DICOTÓMICAS ${ }^{55}$ QUE INDICAN MEIY EMPAREJAMIENTO SELECTIVO, PARA EL NIVEL DE EDUCACIÓN SUPERIOR

\begin{tabular}{lcc}
\hline Variables & $(1)$ & $(2)$ \\
\hline Padre o madre sin educ. y padre o madre con & & 0,0808 \\
educ. básica & & 0,1345 \\
P. o m. sin educ. y p. o m. con educ. media & 0,1437 \\
P. y m. con educ. básica & 0,2298 \\
P. y m. con educ. media & 0,2755 \\
P. o m. sin educ. y p. o m. con educ. superior & & 0,3113 \\
P. o m. con básica y p. o m. con educ. media & & 0,5446 \\
P. o m. con básica y p. o m. con educ. superior & & 0,5834 \\
P. o m. con media y p. o m. con educ. superior & & 0,6705 \\
P. y m. con educ. superior & & 0,0021 \\
Sexo (Hombre = 1) & 0,0015 & 0,0099 \\
Edad & 0,0175 & $-0,0001$ \\
Edad*Edad & $-0,0002$ & $-0,0094$ \\
Tiene pareja (Si = 1) & $-0,0106$ & 0,0284 \\
Zona (Urbano = 1) & 0,0585 & $-0,0034$ \\
Número de personas en el hogar & $-0,0070$ & 0,28 \\
\hline R2 & 0,17 & \\
\hline
\end{tabular}

Fuente: Elaboración del autor en base a encuesta Casen 2013. Nota: Todas las variables son significativas al 0,1 por ciento.

${ }^{55}$ La categoría base es madre y padre sin educación. 
Tabla A.4. ESTIMACIÓN DEL INGRESO DE LA OCUPACIÓN PRINCIPAL PARA HOMBRES (MCO) Y MUJERES (MÉTODO DE HECKMAN)

\begin{tabular}{|c|c|c|c|}
\hline Variables & Hombres & $\begin{array}{c}\text { Mujeres } \\
\text { (ecuación salarios) }\end{array}$ & $\begin{array}{c}\text { Mujeres } \\
\text { (ec. participación) }\end{array}$ \\
\hline Educación básica & $0.186^{\star * *}$ & $0.132^{* * *}$ & -0.0448 \\
\hline Educación media & $0.449^{* * *}$ & $0.421^{\star \star *}$ & $0.298^{* * *}$ \\
\hline Educación superior & $1.177^{* * *}$ & $1.187^{* \star *}$ & $0.934^{* * *}$ \\
\hline Experiencia & $0.0322^{* * *}$ & $0.0312^{* * *}$ & $0.0817^{* * *}$ \\
\hline Experiencia ${ }^{\star}$ Experiencia & $-0.000450^{\star \star *}$ & $-0.000510^{* * *}$ & $-0.00149^{* \star *}$ \\
\hline Tiene Pareja $(1=\mathrm{Si})$ & - & - & $0.0791^{* \star *}$ \\
\hline $\begin{array}{l}\text { Padre o madre sin educ. } \\
\text { y padre o madre con } \\
\text { educ. básica }\end{array}$ & $0.0385^{*}$ & $0.0636^{\star * *}$ & 0.0256 \\
\hline $\begin{array}{l}\text { P. o m. sin educ. y p. o } \\
\text { m. con educ. media }\end{array}$ & $0.0726^{* * *}$ & $0.0940^{* * *}$ & $0.0867^{\star *}$ \\
\hline P. y m. con educ. básica & $0.0845^{* * *}$ & $0.0859^{* *}$ & $0.0858^{* *}$ \\
\hline $\begin{array}{l}\text { P. o m. sin educ. y p. o } \\
\text { m. con educ. superior }\end{array}$ & $0.106^{\star \star *}$ & $0.131^{* * *}$ & $0.105^{\star \star *}$ \\
\hline $\begin{array}{l}\text { P. o m. con educ. básica } \\
\text { y p. o m. con educ. } \\
\text { media }\end{array}$ & $0.183^{* * *}$ & $0.236^{* * *}$ & -0.202 \\
\hline P. y m. con educ. media & 0.0644 & $0.228^{* * *}$ & -0.00564 \\
\hline $\begin{array}{l}\text { P. o m. con educ. básica } \\
\text { y p. o m. con superior }\end{array}$ & $0.206^{* * *}$ & $0.158^{\star * \star}$ & -0.0132 \\
\hline $\begin{array}{l}\text { P. o m. con educ. media } \\
\text { y p. o m. con educ. } \\
\text { superior }\end{array}$ & $0.264^{\star * *}$ & $0.371^{* * *}$ & $0.225^{* * *}$ \\
\hline $\begin{array}{l}\text { P. y m. con educ. su- } \\
\text { perior }\end{array}$ & $0.412^{\star \star \star}$ & $0.505^{* * *}$ & $-0.344^{* * *}$ \\
\hline Constante & $11.89^{* \star *}$ & $11.41^{* \star *}$ & $-0.960^{* * *}$ \\
\hline Tamaño de la muestra & 50,723 & 91,154 & \\
\hline $\mathrm{R} 2$ & 0.31 & - & - \\
\hline
\end{tabular}

Fuente: Elaboración propia en base a encuesta Casen 2013.

Nota: ${ }^{*} p<0,1,{ }^{* *} p<0,05,{ }^{* * *} p<0,01$. 


\section{REFERENCIAS}

Becker, G. 1973. “A Theory of Marriage: Part I”. Journal of Political Economy 81 (4): 813-846.

Beyer, H. 2011. “¿Que veinte años no es nada...? Una mirada a la desigualdad de ingresos a partir de las Encuestas Casen”. Estudios Públicos 121: 5-33.

Brain, I., G. Cubillos \& F. Sabatini. 2007. "Integración social urbana en la nueva política habitacional". Temas de la agenda pública, año 2 (7). Pontificia Universidad Católica de Chile.

Breen, R. \& S. Andersen. 2012. "Educational Assortative Mating and Income Inequality in Denmark". Demography 49: 867-887.

Buss, D. 1985. "Human Mate Selection: Opposites Are Sometimes Said to Attract, but in Fact We Are Likely to Marry Someone Who is Similar to Us in Almost Every Variable". American Scientist 73 (1): 47-51.

Chade, H. \& G. Ventura. 2002. "Taxes and Marriage: A Two-Sided Search Analysis". International Economic Review 43 (3): 955-985.

Eika, L., M. Mogstad \& B. Zafar. 2014. "Educational Assortative Mating and Household Income Inequality". Staff Report 682. Federal Reserve Bank of New York.

Elacqua, G. \& H. Santos. 2013. "Los efectos de la elección escolar en la segregación socioeconómica en Chile: Un análisis georreferenciado". Documento de Referencia 1. Espacio Público.

Fernández, R., N. Guner, \& J. Knowels. 2005. "Love and Money: A Theoretical and Empirical Analysis of Household Sorting and Inequality". The Quarterly Journal of Economics 120 (1): 273-344.

Ferrer, A. \& C. Riddell. 2002. "Sheepskin Effects and the Returns to Education". En Towards Evidence-Based Policy for Canadian Education, editado por Patrice de Broucker \& Arthur Sweetman. Montreal y Kingston: McGillQueen's Press.

Frankel, A. 2014. "Taxation of Couples under Assortative Mating". American Economic Journal: Economic Policy 6 (3): 155-177.

Gaviria, A. 2007. "Social Mobility \& Preferences for Redistribution in Latin America”. Economía 8 (1): 55-96.

González, R. \& B. Mackenna. 2015. "Cuando el éxito personal no se traduce en movilidad social: el caso de los 'triunfadores frustrados' en Chile". Documento de trabajo. http://www.academia.edu/12048264/Cuando_ el_\%C3\%A9xito_personal_no_se_traduce_en_movilidad_social_el_caso_de_ los_triunfadores_frustrados_en_Chile

Greenwood, J., N. Guner, G. Kocharkov \& C. Santos. 2014. "Marry Your Like: Assortative Mating and Income Inequality". American Economic Review 104 (5): 348-53.

Heckman, J. 1979. "Sample Selection Bias as a Specification Error". Econometrica 47 (1): 153-161. 
Hungerford, T. \& G. Solon. 1987. "Sheepskin Effects in the Returns to Education". Review of Economics and Statistics 69 (1): 175-177.

Jaeger, D. \& M. Page. 1996. "Degrees Matter: New Evidence on Sheepskin Effects in the Returns to Education". Review of Economics and Statistics 78 (4): 733 740 .

Mincer, J. 1974. Schooling, Experience and Earnings. New York: National

Bureau of Economic Research.

Núñez, J. \& L. Miranda. 2007. "Recent Findings on Intergenerational Income and Educational Mobility in Chile". Serie Documentos de Trabajo 244. Departamento de Economía, Universidad de Chile.

Siow, A. 2015. "Testing Becker's Theory of Positive Assortative Matching." Journal of Labor Economics 33 (2): 409-441.

Torche, F. 2010. "Educational Assortative Mating and Economic Inequality: A Comparative Analysis of Three Latin American Countries". Demography 47 (2): 481-502.

Torche, F. 2005. "Unequal but Fluid: Social Mobility in Chile in Comparative Perspective”. American Sociological Review 70 (3): 422-450.

Valenzuela, J. \& S. Duryea. 2011. "Examinando la prominente posición de Chile a nivel mundial en cuanto a desigualdad de ingresos: comparaciones regionales". Estudios de Economía 38 (1): 259-293.

Warren, B. 1966. "A Multiple Variable Approach to the Assortative Mating Phenomenon”. Eugenics Quarterly 13 (4): 285-290. EP 
